\title{
A Computational and Geometric Approach to Phase Resetting Curves and Surfaces*
}

\author{
Antoni Guillamon ${ }^{\dagger}$ and Gemma Huguet ${ }^{\ddagger}$
}

\begin{abstract}
This work arises from the purpose of applying new tools in dynamical systems to time problems in biological systems. The main aim of this paper is to develop a numerical method to perform the effective computation of the phase advancement when we stimulate an oscillator which has not yet reached the asymptotic state (a limit cycle). That is, we want to extend the computation of the phase resetting curves (PRCs) (the classical tool to compute the phase advancement) to a neighborhood of the limit cycle, obtaining what we call the phase resetting surfaces (PRSs). To achieve this goal we first perform a careful study of the theoretical grounds (the parameterization method for invariant manifolds and another approach using Lie symmetries), which allows us to describe the isochronous sections of the limit cycle and, from them, to obtain the PRSs. In order to make this theoretical framework applicable, we use the numerical algorithms of the parameterization method and other semianalytical tools to extend invariant manifolds; as a result, we design a numerical scheme to compute both the isochrons and the PRSs of a given oscillator. Finally, to illustrate this algorithm, we apply it to some well-known biological models and we include a discussion on different biological and numerical aspects suggested by these examples.
\end{abstract}

Key words. parameterization method, Lie symmetries, isochrons, phase resetting curves, numerical computation of invariant objects, biological oscillators

AMS subject classifications. 34C14, 34C20, 92-08, 37N25, 92B05

DOI. $10.1137 / 080737666$

1. Introduction. The behavior of coupled oscillators in biology and, more intensively, in neuroscience has been the subject of a great deal of recent interest, and there is a wide literature on this topic (see [17] for a survey), mainly because many oscillators can be described by their phase variables. Moreover, under generic conditions, the phase of the oscillation can also be defined outside the hyperbolic limit cycle via asymptotic phase. Thus, the stable manifold of a point $x_{0}$ on a limit cycle is the union of points having equal phases, and it is often referred to as the isochron of $x_{0}$.

To study synchronization, a useful measurable property of a neural oscillator is its phase resetting curve (PRC). The PRC is found by perturbing the oscillation with a brief stimulus at different times on its cycle and measuring the resulting phase-shift from the unperturbed

\footnotetext{
${ }^{*}$ Received by the editors October 9, 2008; accepted for publication (in revised form) by D. Terman May 26, 2009; published electronically August 19, 2009. This work was partially supported by the MCyT/FEDER grant MTM2006-00478 (DACOBIA) and Generalitat de Catalunya grant 2005SGR-986.

http://www.siam.org/journals/siads/8-3/73766.html

${ }^{\dagger}$ Dept. de Matemàtica Aplicada I, Universitat Politècnica de Catalunya, Dr. Marañón 44-50, E-08028, Barcelona, Catalonia (antoni.guillamon@upc.edu).

‡Centre de Recerca Matemàtica, Apartat 50, E-08193, Bellaterra (Barcelona), Catalonia (gemma.huguet@upc. edu). The work of this author was supported by the Spanish fellowship AP2003-3411 and the NSF grant DMS 0354567.
}

1005

Copyright ( $)$ by SIAM. Unauthorized reproduction of this article is prohibited. 
system. It is a very useful tool to explain how the coupling between neurons can affect the phase and lead them to synchronized or nonsynchronized activity.

PRCs constitute a powerful resource in time-control problems in biological processes. For instance, in the study of circadian rhythms, PRCs are indicators for the experimentalists to know the peaks of the phase advancement and for the practitioners to administrate drugs (see, for instance, [4], [6], or [23] for different contexts), that is, to know the optimal phase advancement.

Different methods of computing the PRCs are known; see [17, Ch. 10] for a survey. One of the most effective is the so-called adjoint method; see [9] (see also [2] for a review). Recently, Govaerts and Sautois (see [11]) have developed a new algorithm to solve the adjoint method problem accompanied with the implementation of continuation methods to study PRCs along families of vector fields with a persistent limit cycle.

Typically, solutions to the models of interest tend asymptotically to a limit cycle. However, one may be interested in computing the phase advancement in the transient state, when the dynamics has not relaxed back to the limit cycle. This occurs when the period of stimulation is too short and is favored by factors like a slow attraction to the limit cycle, a large stimulus amplitude, other external stimuli, random fluctuations, and bursting-like stimuli. Thus, the study of the phase advancement under a certain stimulus in a neighborhood of the limit cycle, not only on the limit cycle, is also interesting.

In this paper, we present a numerical method to extend the computation of the PRCs to a neighborhood of the limit cycle, thus obtaining what we call phase resetting surfaces (PRSs). Therefore, we can evaluate the phase advancement even when the stimulus is applied when the state variables are out of the limit cycle. The results are achieved by means of two steps: studying the theoretical basis describing the isochrons and PRSs (section 3) and developing a numerical procedure that makes this theoretical framework applicable (section 4).

The mathematical formalism is based on the so-called parameterization method, which is a method to parameterize invariant manifolds around an invariant object (see, for instance, [3]); in particular, it provides a parameterization of the isochrons of a limit cycle $\gamma$ of a given vector field $X$. Another approach to obtaining the isochrons is to use that they are the orbits of a vector field $Y$ satisfying a Lie symmetry; that is, $[Y, X]=\mu Y$ (see [25]). In Theorem 3.1, we show the equivalence between these two approaches and, in Proposition 3.6, we use the latter to define the PRSs consistently.

Computational aspects play a major role since the examples of interest are far from being explicitly solvable. The numerical scheme relies on the implementation of the parameterization method (much easier to implement than the computation of Lie symmetries), which provides a local approximation of the isochrons and PRSs (see Steps 1-4 in section 4). After that, we globalize them to a bigger domain by adapting refined methods to globalize invariant manifolds (see [27]) and using the theoretical results of Proposition 3.6 (see Step 5 in section 4).

In the examples, we also include a discussion on the relationship between the excitability types and the types of the corresponding PRCs. This issue was introduced by Ermentrout in [8]; models with strictly positive or mainly positive PRCs are usually called "Type I PRC" or "Class I," whereas models whose PRCs change sign and present a negative regime (delay in the phase) are known as "Type II PRC" or "Class II." The PRC types have effects on the synchronization of an oscillator with a periodic pulse train. For instance, Type I

Copyright (c) by SIAM. Unauthorized reproduction of this article is prohibited. 
models, that is, those with PRCs mostly positive, easily synchronize with fast inputs, but they cannot synchronize with slower inputs. This is because they can advance the phase to catch up with faster inputs, but they cannot delay the phase. This is not the case for Type II models because they can advance or delay the phase. For the specific conductance-based model (5.5), by considering two different parameter values, we can observe the evolution from one type to another, in particular, how the negative parts of the PRC for a Type II PRC oscillator $\left(I_{a p p}=165\right)$ shrink as some bifurcation parameter evolves until it almost vanishes near a Type I excitability value $\left(I_{a p p}=10\right.$, close to a frequency zero limit cycle bifurcation), corresponding also to a Type I PRC value. However, we are more concerned with the observation and the biological consequences of the PRSs rather than carrying out a systematic computation of PRCs with respect to some parameter.

In the examples we also show (see Figures 3-7) that, depending on the geometry of the isochrons, the shape of the PRS may be different from the shape of the PRC. This fact may induce misestimations of the phase advancement when one takes into account only the information provided by the PRCs.

The paper is organized as follows: in section 2 we give the necessary background (on isochronous sections, Lie symmetries, the parameterization method, and PRCs) to tackle the rest of the paper. In section 3, we give the theoretical grounds of the paper, on one hand relating the Lie symmetries with the parameterization method and on the other proving that the solution that we obtain is also a solution of the classical adjoint equation used to compute PRCs on the limit cycle. To facilitate a reading oriented to the more practical aspects, the proofs of these results are given in the appendices. In section 4 , we divide the implementation into five steps; we expand the details of each step in several subsections. We end the paper with some examples in section 5 and a final discussion in section 6 .

2. Background and statement of the problem. In this section we go through the background on the main tools that will be related later in section 3. In general, these tools are defined for vector fields in $\mathbb{R}^{d}$, although for the purposes of this paper we will restrict our attention to $d=2$ from section 3 on.

2.1. Isochronous sections of a limit cycle. Let us consider an autonomous system of ODEs

$$
\dot{x}=X(x), \quad x \in U \subseteq \mathbb{R}^{d}, d \geq 2,
$$

having a periodic orbit $\gamma$ of period $T$, parameterized by $\theta=t / T$ as

$$
\begin{aligned}
\gamma: \mathbb{T}:=\mathbb{R} / \mathbb{Z} & \rightarrow \mathbb{R}^{d}, \\
\theta & \mapsto \gamma(\theta)
\end{aligned}
$$

in order to have period 1 , that is, $\gamma(\theta)=\gamma(\theta+1)$.

For the numerical purposes of this paper we will assume that $X$ is an analytic vector field. Nevertheless, in some cases the theoretical background that we are quoting in this section is still valid for lower regularity vector fields.

Definition 2.1. We say that a point $q \in \Omega \subset \mathbb{R}^{d}$, where $\Omega$ is an open domain containing the limit cycle $\gamma$, is in asymptotic phase with a point $p \in \gamma$ if 


$$
\begin{gathered}
\lim _{t \rightarrow+\infty}\left|\phi_{t}(q)-\phi_{t}(p)\right|=0 \quad \text { or } \\
\lim _{t \rightarrow-\infty}\left|\phi_{t}(q)-\phi_{t}(p)\right|=0,
\end{gathered}
$$

where $\phi_{t}$ is the flow associated to the vector field $X$.

The set of points having the same asymptotic phase is called the isochron.

Definition 2.2. We will say that a limit cycle $\gamma$ is isochronous if there exists an open neighborhood $\Omega$ containing $\gamma$ such that every point in $\Omega$ is in phase with a point on $\gamma$.

Remark 2.3. Notice that the isochrons are mapped to isochrons by the flow $\phi_{t}$ of the vector field $X$. Hence, they are $\phi_{T}$-invariant; that is, $\phi_{T}(q)$ belongs to the isochron of $q$.

This extends the notion of phase of oscillation to a neighborhood in the basin of attraction of the limit cycle. Hence, in a neighborhood $\Omega$ of the limit cycle $\gamma$ there exists a unique scalar function

$$
\begin{aligned}
\vartheta: \Omega \subset \mathbb{R}^{d} & \rightarrow \mathbb{T}=[0,1), \\
x & \mapsto \vartheta(x)
\end{aligned}
$$

such that

$$
\lim _{t \rightarrow+\infty(-\infty)}\left|\phi_{t}(x)-\gamma(\vartheta(x)+t / T)\right|=0 .
$$

The value $\vartheta(x)$ is the asymptotic phase of $x$, and the isochrons are the level sets of $\vartheta$, since the phase is constant on each isochron.

2.2. Isochrons, stable manifolds, and Lie symmetries. From a seminal paper by Winfree [28] and the theoretical answers given by Guckenheimer in a subsequent paper [13], it is known that phase sets or isochrons and stable manifolds (see [16]) of hyperbolic limit cycles have a common link: if the limit cycle is stable, then the isochrons are the leaves of the stable manifold; that is, $W^{s}(\gamma(\theta))$ for $\theta \in \mathbb{T}$. Notice that the case of a hyperbolic unstable limit cycle is equivalent to the stable case just reversing the time.

New papers recovering the problem of the existence of isochrons for a generic nonhyperbolic limit cycle in the plane have appeared recently [5], [25]. From Chicone and Liu's work, [5], we know that a limit cycle $\gamma$ of a $\mathcal{C}^{2}$ planar vector field is isochronous if and only if it is either a hyperbolic or a nonhyperbolic limit cycle satisfying $P(\sigma)=\sigma+c \sigma^{m}+o\left(\sigma^{m}\right)$, with $c \neq 0$ and $m \geq 2$, and $\tau^{\prime}(p)=\cdots=\tau^{(m-1)}(p)=0$, where $p \in \gamma, \tau$ is the time of the first return to a Poincaré section $\Sigma$ at $p$ (parameterized by $\sigma$ ), and $P$ is the corresponding Poincaré map. Moreover, in [25], Sabatini proves that a limit cycle $\gamma$ of a $\mathcal{C}^{2}$ planar vector field $X$ is isochronous if and only if the vector field $X$ is an infinitesimal generator of another $C^{2}$ planar vector field $Y$ transversal to $X$, that is, if

$$
[Y, X]=\mu Y
$$

for some $C^{2}$ function $\mu: \mathbb{R}^{2} \longrightarrow \mathbb{R}$, where [,] stands for the Lie bracket of the two vector fields (see [21]). Moreover, it is stated that, in this case, the orbits of $Y$ crossing the limit cycle $\gamma$ are its isochrons because, by Lie symmetry, the flow of $X$ sends orbits of $Y$ to orbits of $Y$. 
Finally, Freire, Gasull, and Guillamon [10] give a closed formula for the characteristic exponent of a limit cycle $\gamma$ of a planar $\mathcal{C}^{1}$ vector field $X$ in terms of $\mu$ in (2.4). More precisely, they prove that the characteristic exponent of $\gamma$ is given by

$$
\lambda=\int_{0}^{T} \mu(\gamma(t / T)) d t .
$$

Although the result of Sabatini is a nice geometrical characterization of isochronous limit cycles, the difficulty arises when trying to find $\mu$ and $Y$. In this paper, we link this result with the parameterization method developed in [3] to compute a parameterization of the twodimensional stable manifold containing $\gamma$, and we implement it numerically to obtain local expansions of $\mu$ and $Y$.

2.3. The parameterization method for flows. In this subsection we describe the parameterization method introduced by Cabré, Fontich, and de la Llave [3]. The main idea of this method is to compute the invariant manifolds of a dynamical system by looking for a parameterization of them in such a way that the dynamics on this manifold expressed in the coordinates of such parameterization writes as simply as possible.

We will first present the method in its most general form, and then we will consider the particular case of the stable invariant manifold of a periodic orbit of a planar system.

Given a vector field $X$ in $U \subset \mathbb{R}^{d}$, the parameterization method consists of looking simultaneously for an embedding $K: \mathcal{U} \subset \mathbb{R}^{n} \rightarrow \mathbb{R}^{d}$ and a vector field $\mathcal{X}$ in $\mathcal{U} \subset \mathbb{R}^{n}, n \leq d$ and $\mathcal{U}$ open, such that

$$
D K \mathcal{X}=X \circ K
$$

which tells us that the $n$-dimensional manifold parameterized by $K$ and defined by

$$
\mathcal{M}:=\operatorname{Range}(K)=\left\{K(u) \in \mathbb{R}^{d} \mid u \in \mathcal{U} \subset \mathbb{R}^{n}\right\}
$$

is invariant under the flow of $X$. Moreover, the vector field $\mathcal{X}$ describes the dynamics of the vector field $X$ on the invariant manifold $\mathcal{M}$ in the variable $u \in \mathbb{R}^{n}$, parameterizing $\mathcal{M}$.

When $d=n$, we will say that the vector field $X$ is conjugate to $\mathcal{X}$, i.e., $\mathcal{X}=K^{-1} \circ X \circ K$.

The unknowns in the functional equation given in (2.6) are $K$ and $\mathcal{X}$, and therefore it cannot be solved uniquely, since it is underdetermined. In order to overcome this situation, the parameterization method fixes $\mathcal{X}$ (the dynamics) and looks for a $K$ (a parameterization) satisfying the invariance equation (2.6). The way to choose $\mathcal{X}$ is so that the dynamics on $\mathcal{M}$ is as simple as possible, which relates to the theory of normal forms.

We restrict ourselves now to the case of a planar system (2.1) with $d=2$, which has a hyperbolic stable periodic orbit $\gamma$ (the unstable case is equivalent to just reversing the time) parameterized by the phase $\theta$ as in (2.2). Thus, we are interested in looking for a parameterization $K$ of the two-dimensional stable manifold $\mathcal{M}$ of the periodic orbit $\gamma$. Since $n=d=2$, our problem is equivalent to looking for a change of variables $K$ that conjugates the vector field $X$ to a vector field $\mathcal{X}$ with a simpler expression of the dynamics. In this context, it is natural to parameterize it in terms of the phase variable $\theta$ on the limit cycle and another variable $\sigma$ which moves along a transversal direction to the limit cycle, and to impose

Copyright $\odot$ by SIAM. Unauthorized reproduction of this article is prohibited. 
that the motion generated by the vector field $X$ on $\mathcal{U} \subset \mathbb{R}^{2}$ expressed in the variables $(\theta, \sigma)$ is given by

$$
\left\{\begin{array}{l}
\dot{\theta}=1 / T \\
\dot{\sigma}=\lambda \sigma / T
\end{array}\right.
$$

where $T$ is the period of the limit cycle $\gamma$ and $\lambda$ is the characteristic exponent of $\gamma$; since it is hyperbolic stable, we have that $\lambda<0$. From (2.7), it is straightforward to see that the orbit of any point $q=K\left(\theta_{0}, \sigma\right)$ approaches the orbit of the point $p=K\left(\theta_{0}, 0\right)=\gamma\left(\theta_{0}\right)$ on the limit cycle, and therefore $q$ belongs to the isochron of $p$. Thus, the variable $\sigma$ parameterizes every isochron.

The expression of the vector field $X$ in the variables $(\theta, \sigma)$ can be considered as the normal form for a planar vector field around a hyperbolic limit cycle, reminiscent of the action-angle variables for conservative systems.

Once we have fixed the dynamics in (2.7), which is given by the vector field,

$$
\mathcal{X}=\frac{1}{T} \partial_{\theta}+\frac{\lambda \sigma}{T} \partial_{\sigma}
$$

the problem consists of looking for a map $K$

$$
\begin{aligned}
K: \mathcal{N} \subset \mathbb{T} \times \mathbb{R} & \rightarrow \mathbb{R}^{2}, \\
(\theta, \sigma) & \mapsto K(\theta, \sigma),
\end{aligned}
$$

where $\mathcal{N}$ is an open set such that $\mathbb{T} \times\{0\} \subset \mathcal{N}$, and $K$ satisfies the invariance equation (2.6), which in this case has the form

$$
\left(\frac{1}{T} \partial_{\theta}+\frac{\lambda \sigma}{T} \partial_{\sigma}\right) K(\theta, \sigma)=X(K(\theta, \sigma))
$$

The existence of such a map $K$ is ensured, in the hyperbolic case $(\lambda<0)$, by the stable manifold theorem (see [16]). In Theorem 5.4 in [3], the authors present a proof of a more general result, which includes our particular case of the stable manifold of a periodic orbit of a planar system. It essentially consists of first looking for a solution of the invariance equation (2.9) in terms of formal power series and then showing that this power series indeed converges and defines an analytic function. In section 4.1.1 we review the basic steps of the proof which provide a procedure that leads to practical numerical algorithms. These algorithms are the ones that we use to compute $K$ locally in a neighborhood of the limit cycle. Similar implementations of this method have been performed in [15] for the computation of stable and unstable manifolds of invariant tori in quasi-periodic maps.

A more marginal but interesting case is when $\gamma$ is a parabolic isochronous limit cycle; that is, $\lambda=0$ but has a system of isochrons around it (necessary and sufficient conditions are given in [5]). In this case, one cannot conjugate the dynamics to the linearization around the limit cycle as in the hyperbolic case, because the linear part does not contain information on the stability of the limit cycle, that is, on the dynamics of the variable $\sigma$. Thus, we need to consider higher order terms in the dynamical equation for $\sigma$, but we can still impose the 
dynamics for $\theta$ to be linear. Therefore, one can find a map $K$ that conjugates the dynamics of a vector field to

$$
\left\{\begin{array}{l}
\dot{\theta}=1 / T \\
\dot{\sigma}=F(\theta, \sigma),
\end{array}\right.
$$

where $F$ is a nonvanishing function satisfying $F(\theta, 0)=0$. As in the hyperbolic case, one could express $F(\theta, \sigma)$ in a simplified way, but this is outside the scope of this paper.

2.4. Phase response curves and surfaces. As mentioned in the introduction, PRCs are a key tool to study phase advancement in oscillators. Here we introduce the basic background.

Let us consider an oscillator of the form (2.1) with a stable limit cycle $\gamma$ of period $T$ (let us say, for instance, a periodically spiking neuron) which is stimulated at a phase $\theta=t_{s} / T$ with an arbitrary perturbation.

The effect of the perturbation is to produce a phase shift that can be an advance or a delay depending on the time of the stimulus $t_{s}$ relative to the phase of the oscillation $\theta$, leading to a change of the period. The representation of this phase shift is usually called the phase response curve or phase resetting curve (PRC). They are typically defined as

$$
\Delta \vartheta=\left(T-T_{\text {new }}\right) / T,
$$

where $T_{\text {new }}$ is the period for the perturbed limit cycle.

In this paper we will focus on the particular case of infinitesimally small perturbations in duration and amplitude. In this case, the perturbation consists of a pulse that instantaneously displaces the trajectory away from the limit cycle in a certain direction by a certain amplitude. Mathematically, we consider

$$
\dot{x}=X(x)+\epsilon \delta\left(t-t_{s}\right),
$$

where $\epsilon=\left(\epsilon_{1}, \ldots, \epsilon_{d}\right) \in \mathbb{R}^{d}$ and $\delta(t)$ is the Dirac delta function.

When $|\epsilon| \ll 1$, it is common in the theory of weakly coupled neural oscillators [9] to construct the so-called infinitesimal PRC. Using the scalar function $\vartheta$ given in (2.3) that associates to every point in a neighborhood of the limit cycle a phase in $[0,1)$, it is easy to see that the PRC for an instantaneous perturbation as in (2.12) is mathematically equivalent to

$$
\Delta \vartheta(x)=\langle\epsilon, \nabla \vartheta(x)\rangle=\left\langle\epsilon,\left(\frac{\partial \vartheta}{\partial x_{1}}(x), \ldots, \frac{\partial \vartheta}{\partial x_{d}}(x)\right)\right\rangle
$$

for $x \in \gamma$, where $\langle\cdot, \cdot\rangle$ denotes the dot product. See [17, Ch. 10] for the details and historical references.

Note that the pulse in $(2.12)$ can be in any direction in $\mathbb{R}^{d}$. Usually, one studies the PRCs only for the directions given by a vector basis of $\mathbb{R}^{d}$. For instance, for the planar case $(d=2)$, we will consider the PRCs corresponding to the directions $(1,0)$ and $(0,1)$, which will be referred to as $\mathrm{PRC}_{1}$ and $\mathrm{PRC}_{2}$, respectively. For models in neuroscience, one is usually interested only in the PRC for perturbations in the direction of the voltage, that is, $\partial \vartheta(x) / \partial V$ for $x \in \gamma$. When $\epsilon$ is small, abusing language, we will refer to $\Delta \vartheta$ and $\nabla \vartheta$ indistinctly as PRCs. 
Although in the literature the phase shift is computed only on the limit cycle, that is, for $x \in \gamma$, the isochrons allow us to naturally extend it to a neighborhood of the limit cycle and introduce a new concept that we call the phase resetting surface (PRS). In general, PRSs are not considered in the literature because the methods to obtain the PRCs are not easily extendable.

The PRS tabulates the change in the phase produced by a perturbation as a function of the phase $\theta$ and the "distance" $\sigma$ to the limit cycle, computed on the isochron at which it is received. Notice that the PRC is just the section $\sigma=0$ of the PRS. Hence, PRSs are a generalization of the PRCs for $\sigma \neq 0$. This tool can be very useful if we want to stimulate the oscillator repeatedly, without needing to wait for the oscillator to relax back to the limit cycle attractor. This required time to relax is especially inconvenient when the attraction to the limit cycle is too slow or the amplitude of the stimulus is too large.

The classical method for computing PRCs was introduced by Ermentrout and Kopell in [9] and is commonly known as the adjoint method. In section 3.2 we describe a new alternative method that allows us to compute not only the PRC but also the PRS, using the Lie symmetries' formalism and the numerical scheme provided by the parameterization method.

3. Theoretical results. In this section we focus on the case of planar vector fields, and we present the theoretical results that link the concepts presented in section 2, which will allow us to construct a numerical method to simultaneously compute isochrons, PRCs, and PRSs.

3.1. The relation between Lie symmetries and the parameterization method. In this section we establish a relation between the existence of a Lie symmetry (2.4) of the planar vector field $X$ having an isochronous limit cycle and the existence of a map $K$ that conjugates the dynamics of the vector field $X$ to one with the phase variable $\theta$ moving at a constant velocity $1 / T$ (as in (2.7) and (2.10)).

We first state this relation in Theorem 3.1 for the case of a planar vector field having an isochronous limit cycle (without considering whether it is hyperbolic or not), and in Corollary 3.5 we give a more precise result for the hyperbolic case. In section 3.2 we will use this relation to obtain an expression to compute the PRC and the PRS.

Theorem 3.1. Let $X$ be a planar analytic vector field with a limit cycle $\gamma$ of period $T$ parameterized by $\theta$ according to (2.2). Then, the following statements are equivalent:

1. The limit cycle $\gamma$ is isochronous and the isochrons are smooth curves.

2. There exist a transversal vector field $Y$ and a scalar function $\mu$, such that in a neighborhood $\Omega$ of the periodic orbit $\gamma$

$$
[Y, X]=\mu Y .
$$

3. There exist a smooth function $F: \mathbb{T} \times \mathbb{R} \rightarrow \mathbb{R}$ such that $F(\theta, 0)=0$ for all $\theta \in \mathbb{T}$ and a smooth function $K: \mathcal{N} \subset \mathbb{T} \times \mathbb{R} \rightarrow \mathbb{R}^{2}$, where $\mathcal{N}$ is an open set containing $\mathbb{T} \times\{0\}$, such that

$$
\left(\frac{1}{T} \partial_{\theta}+F(\theta, \sigma) \partial_{\sigma}\right) K(\theta, \sigma)=X(K(\theta, \sigma))
$$

Copyright $\odot$ by SIAM. Unauthorized reproduction of this article is prohibited. 
in $\mathcal{N}$; i.e., the vector field $X$ is conjugated to

$$
\mathcal{X}=\left(\frac{1}{T} \partial_{\theta}+F(\theta, \sigma) \partial_{\sigma}\right)
$$

in $\mathcal{N}$.

Moreover, $Y \circ K=\partial_{\sigma} K$ and $\mu \circ K=\partial_{\sigma} F$, or, equivalently,

$$
K(\theta, \sigma)=\psi_{\sigma}(\gamma(\theta))
$$

where $\psi_{\sigma}$ is the flow of the vector field $Y$ and

$$
F(\theta, \sigma)=\int_{0}^{\sigma} \mu(K(\theta, \tau)) d \tau
$$

Proof. See Appendix A.

Before stating a more precise result for the case of hyperbolic limit cycles (which are always isochronous) we would like to make several comments on this theorem.

Remark 3.2. Notice that nothing precise is said about the regularity of the different objects in Theorem 3.1 in terms of the regularity of the vector field X. Although in the case of $\gamma$ being a hyperbolic limit cycle the analyticity of $X$ implies the analyticity of these objects, this is not generally true in the nonhyperbolic case. Then, for the purposes of this paper it will be enough to know that they are regular enough to be defined.

Remark 3.3. The main idea of Theorem 3.1 is that when we have an isochronous limit cycle $\gamma$ of a planar vector field $X$, we can parameterize a neighborhood of the limit cycle in the variables $\theta$, which is the phase of the periodic orbit, and $\sigma$, which is the integration time along the orbits of the vector field $Y$ (isochrons of $X$ ) satisfying (2.4). Moreover, it provides an expression (3.2) for the vector field $X$ expressed in the variables $(\theta, \sigma)$.

Remark 3.4. Notice that the dynamics for $\sigma$ depends on the scalar function $\mu$ (related to $F$ ), whereas the vector field $Y$ (related to the change of coordinates $K$ ) contains information about the geometry of the isochrons.

In section 3.2, we will see that we can obtain the phase advancement on a point in a neighborhood of a limit cycle if we know the vector field $Y$ on this point. In general, given a vector field $X$, it is difficult to find a pair $(Y, \mu)$ satisfying $(2.4)$, except for some academic examples such as those explained in Examples 5.1 and 5.2. However, computing the change of coordinates $K$ is more feasible, at least in a neighborhood of the limit cycle (as we will see in section 4.1). From it, one can easily obtain the above-mentioned pair $(Y, \mu)$ since $Y(K(\theta, \sigma))=\partial_{\sigma} K(\theta, \sigma)$; that is, the motion generated by the vector field $Y$ expressed in the variables $(\theta, \sigma)$ is given by

$$
\begin{aligned}
\dot{\theta} & =0, \\
\dot{\sigma} & =1,
\end{aligned}
$$

and $\mu(K(\theta, \sigma))=\partial_{\sigma} F(\theta, \sigma)$. Of course, the inverse of the map $K$ allows us to recuperate the expression of $Y$ and $\mu$ in the original variables.

Notice that the vector field $Y$ and the scalar function $\mu$ (resp., the map $K$ and the function $F$ ) are not unique. Hence, it is natural to choose them so that the expression of $F$ (or $\mu$ )

Copyright $\odot$ by SIAM. Unauthorized reproduction of this article is prohibited. 
is as simple as possible. We have already seen in section 2.3 where we have reviewed the parameterization method (see also Theorem 5.4 in [3]) that if the limit cycle $\gamma$ is hyperbolic, one can always find an analytical function $K$ so that the function $F$ in $(3.1)$ is $F(\theta, \sigma)=\lambda \sigma / T$ (and therefore $\mu(K(\theta, \sigma))=\lambda / T)$. Hence, the next corollary follows from Theorem 3.1.

Corollary 3.5. Let $X$ be a planar analytic vector field with a hyperbolic limit cycle $\gamma$ of period $T$ and characteristic exponent $\lambda$, parameterized by $\theta$ according to (2.2). Then, the following statements hold and are equivalent:

1. The limit cycle $\gamma$ is isochronous and the isochrons are analytic curves.

2. There exists an analytical vector field $Y$ such that in a neighborhood $\Omega$ of the limit cycle, $\gamma$ is transversal to $X$ and satisfies

$$
[Y, X]=\frac{\lambda}{T} Y
$$

3. There exists an analytical map $K: \mathcal{N} \subset \mathbb{T} \times \mathbb{R} \rightarrow \mathbb{R}^{2}$, where $\mathcal{N}$ is an open set containing $\mathbb{T} \times\{0\}$, such that

$$
\left(\frac{1}{T} \partial_{\theta}+\frac{\lambda}{T} \sigma \partial_{\sigma}\right) K(\theta, \sigma)=X(K(\theta, \sigma))
$$

in $\mathcal{N}$; i.e., the vector field $X$ is conjugated to

$$
\mathcal{X}=\left(\frac{1}{T} \partial_{\theta}+\frac{\lambda}{T} \sigma \partial_{\sigma}\right)
$$

in $\mathcal{N}$.

Moreover, $Y \circ K=\partial_{\sigma} K$, or, equivalently,

$$
K(\theta, \sigma)=\psi_{\sigma}(\gamma(\theta))
$$

where $\psi_{\sigma}$ is the flow of the vector field $Y$.

Proof. The proof follows straightforwardly from Theorem 5.4 in [3] and Theorem 3.1.

Thanks to this reduction, when computing isochrons around a hyperbolic limit cycle we will always use (3.5).

3.2. Computation of isochrons, PRCs, and PRSs. The parameterization $K$ and the vector field $Y$ jointly with the characteristic exponent $\lambda$ allow us to compute the isochrons, the PRCs, and the PRSs.

3.2.1. Computing the isochrons. We already mentioned that the orbit of the points given by $K\left(\theta_{0}, \sigma\right)$ for any $\sigma \in \mathcal{I}_{\theta_{0}}:=\left\{\sigma \in \mathbb{R} \mid\left(\theta_{0}, \sigma\right) \in \mathcal{N}\right\}$ approach (exponentially fast in the hyperbolic case) the orbit of the point $K\left(\theta_{0}, 0\right)=\gamma\left(\theta_{0}\right)$. Therefore, a parameterization of the isochron of the point $\gamma\left(\theta_{0}\right)$ is given by the map

$$
\begin{aligned}
K\left(\theta_{0}, \cdot\right): \mathcal{I}_{\theta_{0}} \subset \mathbb{R} & \longrightarrow \mathbb{R}^{2}, \\
\sigma & \longmapsto K\left(\theta_{0}, \sigma\right) .
\end{aligned}
$$

Copyright $\odot$ by SIAM. Unauthorized reproduction of this article is prohibited. 
3.2.2. Computing the PRC and the PRS. We already mentioned in section 2.4 that, from the mathematical point of view, the change of phase due to a pulse stimulation at a point $p=K(\theta, \sigma)$ in a neighborhood $\Omega$ of the limit cycle $\gamma$ is given by

$$
\nabla \vartheta(p)=\left(\frac{\partial \vartheta}{\partial x}(p), \frac{\partial \vartheta}{\partial y}(p)\right)
$$

In order to compute $\nabla \vartheta(p)$ we consider the following argument: on the one hand, the isochrons are given by the level sets of the function $\vartheta: \mathbb{R}^{2} \rightarrow \mathbb{R}$, introduced in (2.3), which associates a phase to each point in a neighborhood of the limit cycle. On the other hand, they are the orbits of any vector field $Y$ satisfying (2.4). Hence, it is clear that $\nabla \vartheta(p)$ has the same direction as $Y^{\perp}(p)$, which corresponds to the vector orthogonal to $Y$ on $p$, where

$$
Y(p)=Y(K(\theta, \sigma))=\partial_{\sigma} K(\theta, \sigma) .
$$

For $\nabla \vartheta(p)$ to be well defined, we need only to add some normalization. Notice that, for an orbit $\phi_{t}(p), p \in \Omega$, where $\phi_{t}$ is the flow of the vector field $X$, we have

$$
\frac{d \vartheta}{d t}\left(\phi_{t}(p)\right)=1 / T
$$

and using that

$$
\frac{d \vartheta}{d t}\left(\phi_{t}(p)\right)=\left\langle\nabla \vartheta\left(\phi_{t}(p)\right), \frac{d}{d t} \phi_{t}(p)\right\rangle=\left\langle\nabla \vartheta\left(\phi_{t}(p)\right), X\left(\phi_{t}(p)\right)\right\rangle
$$

we have that for any $p \in \Omega$, the PRS is given by

$$
\nabla \vartheta(p)=\frac{Y^{\perp}(p)}{T\left\langle Y^{\perp}(p), X(p)\right\rangle},
$$

where $\langle$,$\rangle denotes the dot product.$

The PRC is just the PRS restricted to the points on the limit cycle, that is, $\sigma=0$. Then, for $p=K(\theta, 0) \in \gamma$,

$$
\nabla \vartheta(K(\theta, 0))=\frac{Y^{\perp}(K(\theta, 0))}{T\left\langle Y^{\perp}(K(\theta, 0)), X(K(\theta, 0))\right\rangle},
$$

where $K(\theta, 0)=K_{0}(\theta)=\gamma(\theta)$ and $Y(K(\theta, 0))$ is given by $Y(K(\theta, 0))=\partial_{\sigma} K(\theta, 0)=: K_{1}(\theta)$. Therefore,

$$
\nabla \vartheta(\gamma(\theta))=\frac{K_{1}^{\perp}(\theta)}{T\left\langle K_{1}^{\perp}(\theta), X(\gamma(\theta))\right\rangle} .
$$

3.3. The relation with the adjoint method. As we already mentioned in the introduction, the most common method in neuroscience to compute phase resetting curves is the adjoint method (see [9], [17]). It essentially computes the gradient of the asymptotic phase along the limit cycle $\gamma$, that is, $\nabla \vartheta(\gamma(t / T))$, by looking for a $T$-periodic solution of the equation

$$
\frac{d Q}{d t}=-D X^{T}(\gamma(t / T)) Q
$$


where $D X^{T}(\gamma(t / T))$ is the transpose of the real matrix $D X(\gamma(t / T))$, with the condition

$$
\langle Q(t), X(\gamma(t / T))\rangle=\frac{1}{T},
$$

which in particular must hold for $t=0$.

This procedure has been automated in the program XPPAUT; see [7].

However, when one wants to extend the adjoint problem to a neighborhood of the limit cycle, one cannot impose periodicity conditions. Thus, the problem lacks conditions to be solved uniquely, unless one knows a way to obtain initial conditions. In the context of this paper, though (see section 3.2), the knowledge of a vector field $Y$ satisfying a Lie symmetry (2.4) provides an expression for $\nabla \vartheta$ at any point in a neighborhood of the limit cycle, and therefore an initial condition for the adjoint problem to be solved uniquely.

In the following proposition, we take advantage of this fact and we extend the adjoint method to a neighborhood of the limit cycle. Indeed, we show that one can compute the phase advancement $\nabla \vartheta$ along the orbits of the vector field $X$ by solving a differential equation (the so-called adjoint equation) with the initial condition provided by (3.8).

Proposition 3.6. Let $\gamma$ be an isochronous T-periodic orbit of a planar analytic vector field $X$ parameterized by $\theta$ according to (2.2). Assume that there exists a transversal vector field $Y$ satisfying (2.4) in a neighborhood $\Omega$. Then, the function $\nabla \vartheta$ along the orbits of the vector field $X$ satisfies the adjoint equation

$$
\frac{d Q}{d t}=-D X^{T}\left(\phi_{t}(p)\right) Q
$$

where $\phi_{t}$ is the flow of the vector field $X$, with the initial condition

$$
Q(0)=\frac{Y^{\perp}(p)}{T\left\langle Y^{\perp}(p), X(p)\right\rangle} .
$$

Proof. See Appendix B.

Remark 3.7. It is clear that the classical adjoint method considers $p \in \gamma$, with $\phi_{t}(p)=$ $\gamma(t / T)$ and $\gamma(0)=p$.

Remark 3.8. We will see in section 4, which is devoted to the numerical implementation, that we will obtain a local approximation of the PRS semianalytically in a local domain $\Omega_{l o c} \supset \gamma$ by computing the parameterization $K$ and using formula (3.8). Proposition 3.6 will be especially useful for the numerical computation to extend the PRS in a bigger domain $\Omega \supset \Omega_{l o c}$ since we will globalize the local approximation by just integrating the adjoint equation (3.11) backward (resp., forward) when the limit cycle is attracting (resp., repelling).

4. Methodology and numerical algorithm. In this section we will put together the theoretical results obtained in section 3 to design a numerical method, which constitutes the main goal of this paper, that computes at the same time the isochrons and the PRSs of an analytic given planar vector field $X$ having a hyperbolic limit cycle.

Without loss of generality we will assume that $\gamma$ is stable. We are going to divide the method into several steps. In order to perform each of them, we will use some standard methods/algorithms that we sketch now and that we explain in detail in this section. Precise references are given for each case.

Copyright $\odot$ by SIAM. Unauthorized reproduction of this article is prohibited. 
Step 1. Solve the invariance equation (3.5) formally for $K$. In order to perform this step we follow the algorithm presented in the proof of Theorem 5.4 in [3]. We will look for a formal solution $K$ of the invariance equation (3.5) expressed as a power series in $\sigma$,

$$
K(\theta, \sigma)=\sum_{n=0}^{\infty} K_{n}(\theta) \sigma^{n},
$$

and matching similar coefficients in $\sigma^{n}$ on both sides of (3.5), we will reduce the problem to solving (4.3) for $K_{0}$ (the limit cycle) and $T$, (4.4) for $K_{1}$ and $\lambda$, and (4.5) for $K_{n}$ with $n \geq 2$, which will be introduced later in section 4.1.1.

For the numerical implementation we will truncate the power series at order $L$. This provides an approximate solution $\tilde{K}$ of the invariance equation (3.5).

We refer the reader to section 4.1.1 for a complete description of the algorithm and to sections 4.1.2 and 4.1.3 for the details of the numerical implementation.

Step 2. Determine the domain of accuracy $\Omega_{\text {loc }}$ of the approximate solution $\tilde{K}$ for a given tolerance. Given a fixed tolerance $E$ we compute

$$
\Omega_{l o c}(E):=\{(\theta, \sigma) \in \mathbb{T} \times \mathbb{R} \mid\|D \tilde{K}(\theta, \sigma) \mathcal{X}(\theta, \sigma)-X(\tilde{K}(\theta, \sigma))\|<E\},
$$

where $\|\cdot\|$ is a norm in $\mathbb{R}^{2}$ and $\mathcal{X}$ is given in (3.6), where $\lambda$ and $T$ are computed in Step 1 . The dependence on $E$ of this domain will be suppressed to simplify notation. In section 4.2, we show that $\Omega_{l o c}$ can be obtained by computing a nonvanishing function $\sigma_{0}: \mathbb{T} \mapsto \mathbb{R}^{+}$, such that

$$
(\theta, \sigma) \in \Omega_{l o c} \Leftrightarrow|\sigma|<\sigma_{0}(\theta) .
$$

We remark that $\Omega_{l o c}$ contains the limit cycle $\gamma$.

Step 3. Compute the isochrons in $\Omega_{\text {loc }}$ by restricting $\tilde{K}$ to a fixed $\theta$. This step is straightforward. Observe that the isochron for $\gamma\left(\theta_{0}\right)$ is obtained from $\tilde{K}$ fixing $\theta=\theta_{0}$ and for $|\sigma|<\sigma_{0}\left(\theta_{0}\right)$. We compute the isochron on suitable points determined by the globalizing method in Step 5 (see section 4.3).

Step 4. Compute the PRS in $\Omega_{\text {loc }}$ from (3.8). This step is straightforward, using $Y(K(\theta, \sigma))$ $=\partial_{\sigma} K(\theta, \sigma)$ in (3.8). Since it will be globalized together with the isochrons, it will be computed on the same points in $\Omega_{l o c}$ as those required to globalize the isochrons (see section 4.3).

Step 5. Globalize the isochrons and the PRS to a domain $\Omega \supset \Omega_{l o c}$ by integrating (2.1) and (3.11). Since the isochrons are invariant for the time- $T$ map of the flow of the vector field $X$, one can take several points on the isochron provided by the local approximation in Step 3 and iterate these points backward for the time- $T$ map of the flow $X$. This procedure extends the isochron to a bigger domain $\Omega$. At the same time, one can compute the PRS for the same points on the local approximation of the isochron according to Step 4 and integrate (3.11) backward together with the vector field for time $T$. This extends the PRS to the points on the global isochron. One can repeat this procedure for several isochrons and obtain the isochrons and PRS for a mesh of points in a bigger domain $\Omega$. We refer to this procedure as the globalization of isochrons and PRSs.

In this paper we have chosen a more refined method to perform this globalization, which follows the numerical method proposed in [27]. Using the idea in [27], we select a nonuniform

Copyright $\odot$ by SIAM. Unauthorized reproduction of this article is prohibited. 
mesh of points on the isochron so that the globalization procedure provides points less sparse on the isochron. Moreover, if the limit cycle is strongly stable (that is, $|\lambda|$ is big), it may happen that, when integrating backward the vector field $X$ up to time $T$, the orbits escape very fast far from the limit cycle. For this reason, we will use instead that the isochrons are carried into isochrons by the vector field $X$, and therefore, in order to globalize the isochron of a point $\gamma\left(\theta_{0}\right)$ we will use the backward iterates of the time- $T \Delta \theta$ map of the flow $X$ taking $\Delta \theta=1 / n$ for some $n \in \mathbb{N}$, for several points on the local approximation of the isochron of the point $\gamma\left(\theta_{0}+k \Delta \theta\right)$ for different $k \in \mathbb{N}$. In section 4.3 we describe in detail the method we have used in this paper to globalize the isochrons and the PRS, which combines these two ideas.

In the following sections, we discuss in more detail the procedure of performing Steps 1, 2 , and 5, including some numerical details about the implementation we have carried out. This procedure is based on combining different methods and algorithms already present in the literature, either borrowing them directly or adapting them to our problem. We specify this in each case.

\subsection{Solving the invariance equation (Step 1).}

4.1.1. A formal solution for the invariance equation. In this section, we review the basic steps of the algorithm presented in the proof of Theorem 5.4 in [3] to solve the invariance equation (3.5) and prove the existence of a solution $K$. We refer the reader to [3] for more details and a rigorous proof of the results.

In order to solve the invariance equation (3.5), we will first look for a $K$ as a power series

$$
K(\theta, \sigma)=\sum_{n=0}^{\infty} K_{n}(\theta) \sigma^{n}
$$

where the components of $K_{n}$ are periodic functions of period 1, and then we will match the coefficients in $\sigma^{n}$ on both sides of (2.9).

For $n=0$, one obtains

$$
\frac{1}{T} \frac{d}{d \theta} K_{0}(\theta)=X\left(K_{0}(\theta)\right),
$$

which admits the solution $K_{0}(\theta)=\gamma(\theta)$, where $\gamma$ is a parameterization of the limit cycle given in (2.2) and $T$ is the period of $\gamma$.

Remark 4.1. Notice that if $K_{0}(\theta)$ is a solution, then $K_{0}(\theta+\omega)$ is also a solution for any $\omega \in[0,1)$. Therefore, there is some ambiguity in parameterizing the phase of an oscillation that can be avoided by fixing the initial point corresponding to the zero phase. It can be fixed anywhere on the limit cycle. In the context of tonic spiking in neuroscience, for instance, it is common to fix $\theta=0$ at the peak of the spike.

For $n=1$, we obtain

$$
\frac{1}{T} \frac{d}{d \theta} K_{1}(\theta)+\frac{\lambda}{T} K_{1}(\theta)=D X\left(K_{0}(\theta)\right) K_{1}(\theta),
$$

which tells us that $K_{1}(\theta)$ is an eigenfunction with eigenvalue $-\lambda$ of the operator $\mathcal{L}$ defined by

$$
\mathcal{L}:=\frac{d}{d \theta}-T\left(D X \circ K_{0}\right)(\theta)
$$

Copyright (c) by SIAM. Unauthorized reproduction of this article is prohibited. 
Using Proposition 5.2 in [3], we know that $K_{1}(\theta)$ is a solution of (4.4) with eigenvalue $-\lambda$ if and only if $K_{1}(0)$ is an eigenvector of the monodromy matrix $\Phi_{1}$ with eigenvalue $e^{\lambda}$. The monodromy matrix can be computed by solving the first variational equation

$$
\frac{d}{d \theta} \Phi_{\theta}=T D X\left(K_{0}(\theta)\right) \Phi_{\theta}
$$

with $\Phi_{0}=\mathrm{Id}$, and taking the value $\Phi_{1}$. Of course, now the problem reduces to computing the eigenvalues and eigenvectors of a real matrix.

Recall that for planar vector fields, the other eigenvector is given by the vector field $X\left(K_{0}(0)\right)=X\left(K_{0}(1)\right)$ with associated eigenvalue 1 .

Finally, it is easy to see that $K_{1}(\theta)=e^{-\lambda \theta} \Phi_{\theta} K_{1}(0)$ is a solution of (4.4).

Remark 4.2. For the numerical computations when the eigenvalue $e^{\lambda}$ is very small, we will use that $\lambda=\int_{0}^{T} \operatorname{div}(X(\gamma(t / T))) d t$.

Remark 4.3. Notice that if $K_{1}(\theta)$ is a solution of (4.4), then $b K_{1}(\theta)$ for any $b \in \mathbb{R}$ is also a solution. Even though all the choices of $K_{1}(\theta)$ are mathematically equivalent, the choice affects the numerical properties of the algorithm. See Remark 4.5 for a more detailed discussion.

For $n \geq 2$, we have

$$
\frac{1}{T} \frac{d}{d \theta} K_{n}+\frac{n \lambda}{T} K_{n}=\left(D X \circ K_{0}\right) K_{n}+R_{n}
$$

where $R_{n}$ is an explicit polynomial in $K_{0}, \ldots, K_{n-1}$ whose coefficients are derivatives of $X$ evaluated at $K_{0}$. These coefficients will be computed numerically using the methods of automatic differentiation (see, for instance, [12] and [18]).

By Proposition 5.2 in [3], (4.5) for $n \geq 2$ can be solved provided that $e^{n \lambda}$ is not an eigenvalue of the monodromy matrix $\Phi_{1}$ associated to $\gamma$. Notice that this assumption is satisfied for planar vector fields, provided that the limit cycle is hyperbolic, that is, $\lambda \neq 0$.

Once $K_{0}(\theta)$ and $K_{1}(\theta)$ are fixed (see Remarks 4.1 and 4.3), the solution $K_{n}(\theta)$ for $n \geq 2$ of (4.5) is uniquely determined. Taking into account that $K_{n}$ are periodic solutions in $\theta$, as is usual in numerical implementations, we will discretize (4.5) using Fourier series and reduce the problem to solving a linear system in the Fourier space; see sections 4.1.2 and 4.1.3 for more details.

Finally, by Theorem 5.4 in [3] we know that, provided that $\lambda$ satisfies the mentioned conditions, the series constructed here converges to a true analytic solution of the problem.

For practical numerical implementations, it is clear that one can compute only a finite number of terms in the Taylor expansion. Therefore, we will compute only an approximation up to a certain order $L$ of the real function $K$. Of course, the accuracy of these approximations depends on the number of terms computed in the Taylor expansion as well as the size of the neighborhood of the limit cycle where we evaluate the function. See section 4.2 for a discussion of this aspect.

4.1.2. Fourier-Taylor series. We have seen in the previous section that in order to solve (3.5) it will be convenient to consider Fourier-Taylor expansions of the unknown $K$, which provides the conjugacy. Considering series expansions of the unknowns and computing the coefficients numerically are a classical methodology in celestial mechanics to compute invariant

Copyright $\odot$ by SIAM. Unauthorized reproduction of this article is prohibited. 
manifolds and normal forms. Before discussing how to numerically solve the equations, we review here some basic algorithms to manipulate these series numerically, and we discuss how to store them. We also provide references for the reader interested in further details.

As we already mentioned in section 4.1.1, we first seek $K$ as a power series in $\sigma$,

$$
K(\theta, \sigma)=\sum_{n=0}^{\infty} K_{n}(\theta) \sigma^{n},
$$

where $K_{n}(\theta)$ are 1-periodic functions in $\theta$. Thus, using Fourier formalism, the $K_{n}(\theta)$ can be written as

$$
K_{n}(\theta)=\sum_{k \in \mathbb{Z}} c_{k}^{n} e^{2 \pi \mathrm{i} k \theta} .
$$

Since $K_{n}$ are real functions, we need only to store half of the coefficients or, equivalently, store the cosine and sine Fourier series:

$$
K_{n}(\theta)=a_{0}^{n}+\sum_{k>0} a_{k}^{n} \cos (2 \pi k \theta)+b_{k}^{n} \sin (2 \pi k \theta),
$$

where $a_{0}^{n}=\operatorname{Re}\left(c_{0}^{n}\right), a_{k}^{n}=2 \operatorname{Re}\left(c_{k}^{n}\right)$, and $b_{k}^{n}=-2 \operatorname{Im}\left(c_{k}^{n}\right)$ for $k>0$.

Remark 4.4. One of our goals is to apply this method to classical systems in neuroscience. The main practical shortcoming in these cases is that the Fourier series are not adaptable to the usual presence of spikes (slow-fast systems), where the Fourier coefficients decrease very slowly and not uniformly. Although these systems can be analytic, from this numerical point of view they behave as if they are not. In these cases, other methods of discretization which are more adaptive like splines or wavelets could give some improvements.

We have seen that the discretization of the invariance equation (3.5) using Taylor expansions reduces the problem to solving the set of equations (4.3)-(4.5), which involves the numerical evaluation of derivatives of $X$ at $K_{0}(\theta)$; in particular, to compute the term $R_{n}(\theta)$ in (4.5), we need to evaluate the derivatives up to order $n$. In this context, automatic differentiation is a very suitable tool to perform these computations. It consists of a set of techniques to compute the derivatives of arbitrary order of a function evaluated at a fixed value, accurate to working precision, avoiding in this way the numerical problems inherent in symbolic and numerical differentiation. They are based on writing the function as a sequence of algebraic operations (sum, product, ...) and elementary transcendental functions (exp, sin, cos, log, power, ...), and then applying systematically the chain rule to these operations (see [12], [14], [18], and also the Web page of the automatic differentiation community http://www.autodiff.org/). Since the vector field $X$ is analytic, it can be expressed as a sequence of elementary operations and transcendental functions, and automatic differentiation allows us to evaluate the function $R_{n}$ in (4.5) for $n \geq 2$.

We have also seen that in the whole process we deal with periodic functions, which we can store keeping either $N+1$ Fourier coefficients or the values of the function on a grid of $N$ equidistant points in real space. Depending on the operator acting on them, we will choose the method that is more efficient in terms of the number of operations. Thus, the equations presented in the previous section involve some operators that will be diagonal in Fourier space (for instance, derivatives) and some that will be diagonal in real space (for instance, products

Copyright (c) by SIAM. Unauthorized reproduction of this article is prohibited. 
and composition with transcendental functions). In order to pass from a grid representation to Fourier series and vice versa we use the fast Fourier transform (FFT) (see, for instance, [1] for a classical reference). In this work we have used the fftw3 library (see http://www.fftw.org/).

4.1.3. Numerical computation of $K_{n}$ and estimation of the error. In this section we discuss different methods to numerically solve (4.3), (4.4), and (4.5) for $n \geq 2$ and determine its accuracy.

Observe that (4.3) and (4.4) are special because they involve four unknowns $\left(K_{0}(\theta), T\right.$, $\left.K_{1}(\theta), \lambda\right)$. These two equations will be solved simultaneously using additional information. Instead, (4.5) can be treated the same way for any $n \geq 2$.

In order to solve (4.3) and (4.4) we will integrate the system of ODEs. The integration method used is a Taylor method (we have used the routines provided by Jorba and Zou; see [18] and http://www.maia.ub.es/ angel/soft.html). We used adaptive step size, degree, and a tolerance (absolute and relative) of $10^{-16}$.

Recall that for $n=0$ we need to look for a periodic solution. In order to compute it, we consider a Poincaré section and reduce the problem to finding a zero of the Poincaré map that can be achieved using a Newton method. Note that for the Newton method we will need to integrate the variational equations together with the vector field. The solution for the variational equations will be used to solve (4.4) according to the method explained in section 4.1.1.

Once we obtain the limit cycles $K_{0}(\theta)$ and $K_{1}(\theta)$ we store them for equidistant values of $\theta$; that is, $\theta_{i}=i / N$ for $i=0, \ldots, N-1$. Notice that this is equivalent to storing the coefficients of the Fourier series up to degree $N$ by means of the FFT algorithm.

For $n \geq 2$ the most straightforward method is to discretize (4.5) using a basis of $N+1$ Fourier coefficients and then apply a linear solver. However, once we have obtained $K_{0}$ and $K_{1}$, we can perform a change of coordinates given by $(x, y)=g(\theta, \sigma)=K_{0}(\theta)+\sigma K_{1}(\theta)$. If we apply the method again to the system obtained after this change, then it turns out that (4.5) becomes diagonal in Fourier series. Once we obtain the solution as a Fourier series we can go back to real space using the FFT. Again, as in the previous cases we store $K_{n}$ for equidistant values of $\theta$. An alternative method consists of applying a quasi-Newton method to the invariance equation (see, for instance, [15]). By now, the results shown in section 5 have been obtained using the straightforward method.

In order to decide up to which order $N$ we compute the Fourier series, we require that the residuals are of a size of order smaller than a certain tolerance $E_{\text {tail }}$ that we set at $10^{-12}$. That is, we truncate the Fourier series up to some order $N$ in such a way that the norm of the last $10 \%$ of Fourier coefficients is smaller that the considered order; in symbols

$$
\left|K_{n}^{\text {tail }}\right|=\sum_{k=\lfloor 0.9 N / 2\rfloor}^{N / 2}\left|a_{k}^{n}\right|+\left|b_{k}^{n}\right|<E_{\text {tail }} .
$$

To check the accuracy of the solutions $K_{n}$ obtained, we substitute them in the corresponding equation ((4.3) if $n=0,(4.4)$ if $n=1$, and (4.5) if $n \geq 2)$ for discrete values of $\theta$, that is, $\theta_{i}=i / N$ for $i=0, \ldots, N-1$. For each value $\theta_{i}$, this substitution provides an evaluation of the error $E_{n}\left(\theta_{i}\right)$. Finally, we compute the discrete $\ell_{1}$ norm of $\left\{E_{n}\left(\theta_{i}\right)\right\}_{i=0}^{N-1}$ to get the accuracy; 
that is,

$$
\left\|E_{n}\right\|=\frac{1}{N} \sum_{i=0}^{N-1}\left|E_{n}\left(\theta_{i}\right)\right|
$$

Notice that the computation of $E_{n}\left(\theta_{i}\right)$ again involves an FFT.

4.2. Domain of accuracy of the local approximation (Step 2). Up to this point we assume that we have solved approximately using Fourier series up to degree $N(4.3)$ for $n=0$, (4.4) for $n=1$, and (4.5) for $n=2, \ldots, L$, for a fixed value of $L$, according to the procedure described in the previous sections. Thus, we have an approximate solution of the invariance equation (3.5), which is given by

$$
\tilde{K}(\theta, \sigma)=\sum_{n=0}^{L} \tilde{K}_{n}(\theta) \sigma^{n}
$$

where

$$
\tilde{K}_{n}(\theta)=a_{0}^{n}+\sum_{k=1}^{N / 2} a_{k}^{n} \cos (2 \pi k \theta)+b_{k}^{n} \sin (2 \pi k \theta)
$$

is an approximate solution of (4.3) for $n=0,(4.4)$ for $n=1$, and (4.5) for $n=2, \ldots, L$.

Now, we need to determine the domain $\Omega_{l o c}$ where the solution $\tilde{K}$ is accurate; that is, the function $\tilde{K}$ satisfies the invariance equation (3.5) up to a certain tolerance $E$ that we established as being between $10^{-8}$ and $10^{-12}$ (see definition in (4.1)).

In order to do that, we fix a value of $\theta$, say, $\theta=\theta^{*}$, and we compute $\sigma_{0}\left(\theta^{*}\right)$ satisfying that for all $\sigma \in \mathbb{R}$ such that $|\sigma|<\sigma_{0}\left(\theta^{*}\right)$ one has

$$
\left\|\frac{1}{T} \sum_{n=0}^{L} K_{n}^{\prime}\left(\theta^{*}\right) \sigma^{n}+\frac{\lambda}{T} \sum_{n=0}^{L} n K_{n}\left(\theta^{*}\right) \sigma^{n}-X\left(\sum_{n=0}^{L} K_{n}\left(\theta^{*}\right) \sigma^{n}\right)\right\|<E,
$$

where

$$
K_{n}^{\prime}\left(\theta^{*}\right)=2 \pi \sum_{k=1}^{N / 2} k b_{k}^{n} \cos \left(2 \pi k \theta^{*}\right)-k a_{k}^{n} \sin \left(2 \pi k \theta^{*}\right)
$$

and $\|\cdot\|$ is a norm in $\mathbb{R}^{2}$.

Remark 4.5. Recall that if $K(\theta, \sigma)$ is a solution of the invariance equation (2.9), so is $K(\theta+\omega, b \sigma)$ for any $\omega \in[0,1)$ and $b \in \mathbb{R}$. As we already mentioned in Remark 4.1, the choice of $\omega$ is related to the zero phase for the limit cycle. So, following the usual criterion in neuroscience, we will fix the zero phase for the oscillator at the maximum value of a specific variable, in neuroscience the voltage (spike). The choice of $b$ is related to the domain of convergence. Hence, if we choose a large $b$, the domain where we can evaluate the series will be small. Although mathematically we can choose any value of $b$, for the numerical stability it will be convenient to choose a value of $b$ such that the coefficients $K_{n}$ can be kept at order 1 , so that one can avoid the round-off errors. Notice that if we consider bK$K_{1}$, then the new $K_{n}$ is $b^{n} K_{n}$ for $n \geq 2$.

Copyright ( $\odot$ by SIAM. Unauthorized reproduction of this article is prohibited. 
However, in some cases, the $K_{n}$ are not uniform in $\theta$, and in these cases one cannot find a global $b$. The immediate consequence of this fact is that for some values of $\theta$, the $K_{n}$ become smaller than the machine precision, and one cannot trust them. For these values, increasing the order $L$ of the Taylor polynomial has no effect on increasing the domain where the local approximation is reliable. This phenomenon can be seen in Example 5.5 close to a saddle node of invariant circles (SNIC) bifurcation. See also the section devoted to numerical drawbacks for slow-fast systems in the discussion.

4.3. Globalization method (Step 5). In this section we will describe a method to globalize the isochrons and the PRSs obtained in the domain $\Omega_{l o c}$. Recall that this means extending the isochrons and the PRSs to a bigger domain $\Omega \supset \Omega_{l o c}$, which reaches far away from the limit cycle. The method we propose will be adapted from the one introduced in [27], which incorporates several tricks to improve the standard way to extend local approximations of invariant manifolds.

We will discuss first how to globalize the isochrons, and finally we will see that we can globalize the PRS for the same points obtained on the globalized isochron.

Recall from section 4.2 that, numerically, given an error bound $\left(10^{-8}-10^{-12}\right)$, for each $\theta \in \mathbb{T}$, we can compute the isochron only up to a point $p$ parameterized by $(\theta, \sigma)$ with $|\sigma|<\sigma_{0}(\theta)$. This is the local approximation for the isochron.

Recall also that the isochrons are invariant under the time- $T$ map of the flow $\phi_{t}$ of the vector field $X$, and they lie on the stable manifold. Therefore, a straightforward method to globalize the isochron corresponding to the point $\gamma\left(\theta_{0}\right)$ on the limit cycle involves taking $n$ points on the local isochron, which are parameterized by $\left(\theta_{0}, \sigma\right)$ with $|\sigma| \in\left(\sigma_{0}\left(\theta_{0}\right) e^{\lambda}, \sigma_{0}\left(\theta_{0}\right)\right)$, and then performing iterates of the inverse time- $T$ map $\phi_{-T}$ for these points. This would be the standard way.

However, in many cases (included models in neuroscience in which we are especially interested) the standard method has the disadvantage that we get too many points close to $\gamma\left(\theta_{0}\right)$ and just a few far from it. Moreover, some of them may escape very fast far from the limit cycle.

This last shortcoming can be avoided using that isochrons, even if they are not invariant under the flow of $X$, are preserved by the flow; that is, isochrons are carried onto isochrons. Hence, we can consider inverse time- $T \Delta \theta$ maps $\phi_{-T \Delta \theta}$ taking $\Delta \theta=1 / n, n \in \mathbb{N}$, as well as $n$ local isochrons corresponding to $\gamma\left(\theta_{0}+k \Delta \theta\right)$ for $k=0, \ldots, n-1$. Then, to globalize the isochron corresponding to $\gamma\left(\theta_{0}\right)$, we obtain several points $\left\{p_{i}\right\}_{i=0, \ldots, m}$ on this isochron from points $\left\{q_{i}\right\}_{i=0, \ldots, m}$ on the other isochrons in the following way: consider a point $q_{i}=$ $K\left(\theta_{0}+k_{i} \Delta \theta, \sigma_{i}\right)$ for some $k_{i} \in \mathbb{N}, 0 \leq k_{i} \leq n-1$, and $\sigma_{i} \in \mathbb{R},\left|\sigma_{i}\right| \leq \sigma_{0}\left(\theta_{0}+k_{i} \Delta \theta\right)$, and compute $p_{i}$ as

$$
p_{i}=\phi_{-k_{i} T \Delta \theta}\left(q_{i}\right)
$$

for $i=0, \ldots, m$. See Figure 1 for an schematic illustration of this idea.

The method in [27] (see also [19] for another alternative) provides a systematic way to determine which $\sigma_{i}$ and $k_{i}$ for $i=0, \ldots, m$ one has to choose so that the points $\left\{p_{0}, \ldots, p_{m}\right\}$ obtained on the global isochron are well distributed everywhere on the isochron, overcoming the problem of sparseness far from the limit cycle.

Copyright (c) by SIAM. Unauthorized reproduction of this article is prohibited. 


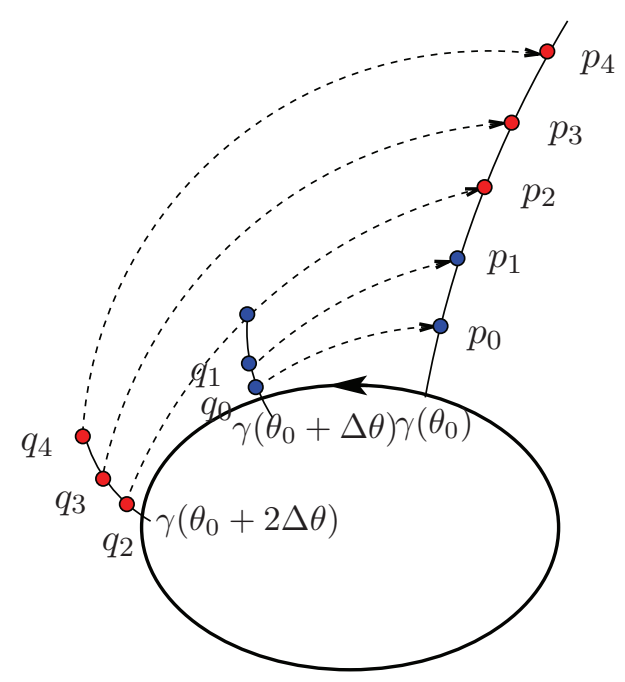
(4.10)

Figure 1. Schematic representation for the globalization procedure with "intermediate" isochrons. See also

Next, we explain in detail the globalization method, which uses the techniques introduced in [27] adapted to the case in which one considers "intermediate" isochrons to globalize them.

We want to extend the local isochron corresponding to a phase $\theta_{0}$. We are going to approximate the isochron by a sequence of points $\left\{p_{0}, \ldots, p_{m}\right\}$ on it, for which we will assume that they are at a distance smaller than some tolerance $\Delta s$, that is,

$$
\left\|p_{m}-p_{m-1}\right\|<\Delta s
$$

where $\|\cdot\|$ is the standard Euclidean norm in $\mathbb{R}^{2}$, and the angle between three consecutive points is bigger than a certain tolerance $\Delta \alpha$,

$$
\left(p_{m-1}-p_{m-2}\right) \cdot\left(p_{m}-p_{m-1}\right) \geq \cos (\Delta \alpha)\left\|p_{m-1}-p_{m-2}\right\|\left\|p_{m}-p_{m-1}\right\| .
$$

Assume that we have computed a sequence of points up to $p_{m}$ satisfying the previous conditions and we have a current value of $\sigma_{m}$ and $\Delta \sigma_{m}$ such that $\sigma_{m}=\sigma_{m-1}+\Delta \sigma_{m}$ and a certain iterate $k$ such that

$$
\phi_{-k T \Delta \theta}\left(K\left(\theta_{0}+k \Delta \theta, \sigma_{m}\right)\right)=p_{m}
$$

We want to predict the new $\sigma_{m+1}$ and therefore $\Delta \sigma_{m+1}$ such that

$$
\phi_{-k T \Delta \theta}\left(K\left(\theta_{0}+k \Delta \theta, \sigma_{m}+\Delta \sigma_{m+1}\right)\right)=p_{m+1},
$$

satisfying that it is at a distance smaller than $\Delta s$ from $p_{m}$.

Then, we consider

$$
\Delta \sigma_{m+1}=\min \left(\frac{\Delta s}{\Delta s_{m}}, \frac{\Delta \alpha}{\Delta \alpha_{m}}, 1.5\right) \Delta \sigma_{m} 0.8
$$

Copyright $\odot$ by SIAM. Unauthorized reproduction of this article is prohibited. 
where $\Delta s_{m}$ is the distance between $p_{m-1}$ and $p_{m}$ and $\Delta \alpha_{m}$ the angle between $\bar{v}=p_{m-1}-p_{m-2}$ and $\bar{w}=p_{m}-p_{m-1}$. The factor 0.8 can be seen as a security factor.

If $\sigma_{m+1}=\sigma_{m}+\Delta \sigma_{m+1}$ falls into the allowed range for $\sigma$, that is, $\left|\sigma_{m+1}\right|<\sigma_{0}\left(\theta_{k}\right)$, where $\theta_{k}=\theta_{0}+k \Delta \theta$, which means that we are in the range where the local approximation of the isochron for $\theta_{k}$ is accurate, we apply the map $\phi_{-k T \Delta \theta}$ and we obtain $p_{m+1}$. In this case we define $k_{m+1}=k$ according to (4.10).

Otherwise, we keep dividing both $\sigma_{m+1}$ and $\Delta \sigma_{m+1}$ by $e^{\lambda \Delta \theta}, l$ times, until $\left|\sigma_{m+1}\right|<$ $\sigma_{0}\left(\theta_{k+l}\right)$. We say then that $k_{m+1}=k+l$ and we compute $p_{m+1}$ from (4.10). Typically $l=1$, but it can be greater. We replace $k$ by $k+l$.

If despite our choice of $\Delta \sigma_{m}$ the obtained point $p_{m+1}$ fails to satisfy one of the conditions, we can either consider a smaller $\Delta \sigma_{m+1}$ (taking into account that the $\Delta \sigma$ 's cannot be smaller than a certain value $\Delta_{\text {min }}$ ) or keep the computed point and use an interpolation method for this part.

Remark 4.6. Of course the map $\phi_{-T \Delta \theta}$ has to be computed by integrating the vector field $X$ backward for time $t=T \Delta \theta$.

Finally, we globalize the PRS in parallel with the isochrons: we approximate them locally according to (3.8) and we globalize them integrating the system (3.11) backward together with the vector field. That is, for each $p_{m}$ obtained from $q_{m}=K\left(\theta_{0}+k_{m} \Delta \theta, \sigma_{m}\right) \in \Omega_{l o c}$ according to (4.10), we compute $\nabla \vartheta\left(p_{m}\right)$ using Proposition 3.6 in the following way:

$$
\nabla \vartheta\left(p_{m}\right)=\Psi_{-k_{m} T \Delta \theta}\left(\nabla \vartheta\left(q_{m}\right)\right)
$$

where $\Psi_{t}$ is the flow of (3.11) and $\nabla \vartheta\left(q_{m}\right)$ is obtained from (3.8).

4.4. Software. The algorithms have been implemented in $\mathrm{C}$ language and have been run under the Linux environment. They have been applied to compute isochronous sections and PRCs of limit cycles for planar vector fields which appear in models of neuroscience and neurobiology.

The program performs the following steps: (1) computation of the limit cycle and its period, the monodromy matrix, and the characteristic exponent; (2) computation of the FourierTaylor expansions of the isochrons; (3) computation of the domain of accuracy and the local approximation for the isochrons and the PRS; (4) globalization of the isochrons and the PRS. The figures are obtained using gnuplot.

Although we already mentioned it in this section, we recall here that we have used a Taylor method to integrate the system of equations and in particular the routines provided by Jorba and Zou in http://www.maia.ub.es/ angel/soft.html. And we have used the fftw3 library to perform the FFT transform.

5. Examples. In this section, we apply our method to representative examples, ranging from the most simple instances of Hopf and SNIC (saddle-node on an invariant curve) bifurcations and the classical van der Pol oscillator to more sophisticated neuronal models. Apart from obtaining isochrons, PRCs, and PRSs, through these examples we want to illustrate different facts: (a) what are the clues to explain the transition from "Type I" PRCs to "Type II" PRCs; (b) the numerical problems that arise when dealing with slow-fast systems; and, (c) up to which degree PRSs show disagreement with PRCs in the same phase and how this can affect high frequency stimulation. We end the paper with a discussion on these facts in section 6 .

Copyright $\odot$ by SIAM. Unauthorized reproduction of this article is prohibited. 
We start with a direct application to the simplest vector fields that exhibit either a Hopf or a SNIC bifurcation, for which we can compute their limit cycle and the corresponding normalizing vector field analytically and we can also get an analytic expression for the PRC.

Example 5.1. We consider a simple example of a supercritical Hopf bifurcation

$$
\left\{\begin{array}{l}
\dot{x}=\beta x-y-x\left(x^{2}+y^{2}\right) \\
\dot{y}=x+\beta y-y\left(x^{2}+y^{2}\right)
\end{array}\right.
$$

for $(x, y) \in \mathbb{R}^{2}$ and $\beta$ being a real parameter, which writes, in polar coordinates $(r, \phi) \in$ $\mathbb{R}^{+} \times[0,2 \pi)$, as

$$
\left\{\begin{array}{l}
\dot{r}=r\left(\beta-r^{2}\right) \\
\dot{\phi}=1
\end{array}\right.
$$

For $\beta=0$, there is a supercritical Hopf bifurcation giving rise, for $\beta>0$, to a stable limit cycle $\gamma$ of radius $\sqrt{\beta}$ and period $T=2 \pi$. We parameterize $\gamma$ by the phase $\theta=\phi /(2 \pi) \in \mathbb{T}$ in the following way:

$$
\gamma(\theta)=(\sqrt{\beta} \cos (2 \pi \theta), \sqrt{\beta} \sin (2 \pi \theta)) .
$$

It is not difficult to see that the vector field $Y(x, y)=(x, y)$ and the function $\mu(x, y)=$ $-2\left(x^{2}+y^{2}\right)$ satisfy the condition (2.4).

Hence, taking into account that $Y^{\perp}=(-y, x)$ and $\left\langle Y^{\perp}, X\right\rangle=x^{2}+y^{2}$, by (3.8) the phase shift for a point $p=(x, y) \in \Omega$ is given by

$$
\nabla \vartheta(p)=\frac{1}{2 \pi}\left(-\frac{y}{x^{2}+y^{2}}, \frac{x}{x^{2}+y^{2}}\right)
$$

Then, using the parameterization of the limit cycle, the PRC is just

$$
\nabla \vartheta(\gamma(\theta))=\frac{1}{2 \pi \beta}(-\sqrt{\beta} \sin (2 \pi \theta), \sqrt{\beta} \cos (2 \pi \theta)) .
$$

That is, $P R C_{1}(\theta)=-\sin (2 \pi \theta) /(2 \pi \sqrt{\beta})$, and $P R C_{2}(\theta)=\cos (2 \pi \theta) /(2 \pi \sqrt{\beta})$.

Example 5.2. The easiest way to obtain a saddle node on an invariant cycle bifurcation is through

$$
\left\{\begin{array}{l}
\dot{r}=r\left(\beta-r^{2}\right) \\
\dot{\phi}=m-\sin (\phi)
\end{array}\right.
$$

where $(r, \phi) \in \mathbb{R}^{+} \times[0,2 \pi)$, and $\beta$ and $m$ are real parameters, which, in cartesian coordinates $(x, y) \in \mathbb{R}^{2}$, writes as

$$
\left\{\begin{array}{l}
\dot{x}=\beta x-m y-x\left(x^{2}+y^{2}\right)+\frac{y^{2}}{\sqrt{x^{2}+y^{2}}} \\
\dot{y}=m x+\beta y-y\left(x^{2}+y^{2}\right)-\frac{x y}{\sqrt{x^{2}+y^{2}}} .
\end{array}\right.
$$

We assume that $\beta>0$ and $m>1$. Therefore, there exists a unique and stable circular limit cycle $\gamma$ of radius $\sqrt{\beta}$ and period $T=2 \pi / \sqrt{m^{2}-1}$ that we parameterize by the phase $\theta$ satisfying $\dot{\theta}=1 / T, \theta \in[0,1)$ in the following way:

$$
\gamma(\theta)=(\sqrt{\beta} \cos (\Omega(\theta)), \sqrt{\beta} \sin (\Omega(\theta))),
$$

Copyright $\odot$ by SIAM. Unauthorized reproduction of this article is prohibited. 
where $\Omega$ is the phase transformation between $\theta$ and $\phi$, given by the solution of the Cauchy problem

$$
\frac{1}{T} \frac{d \Omega}{d \theta}=m-\sin (\Omega(\theta)) ; \quad \Omega(0)=0 .
$$

The explicit solution can be obtained analytically:

$$
\Omega(\theta)=2 \arctan \left(\frac{m \sin (\pi \theta)}{\sqrt{m^{2}-1} \cos (\pi \theta)+\sin (\pi \theta)}\right) .
$$

Again, as in Example 5.1, the vector field $Y(x, y)=(x, y)$ and the function $\mu(x, y)=$ $-2\left(x^{2}+y^{2}\right)$ satisfy condition (2.4).

Hence, taking into account that $Y^{\perp}=(-y, x)$ and

$$
\left\langle Y^{\perp}, X\right\rangle=m\left(x^{2}+y^{2}\right)-y \sqrt{x^{2}+y^{2}},
$$

by (3.8) the PRS for $p=(x, y) \in \Omega$ is given by

$$
\nabla \vartheta(p)=\frac{\sqrt{m^{2}-1}}{2 \pi}\left(-\frac{y}{m\left(x^{2}+y^{2}\right)-y \sqrt{x^{2}+y^{2}}}, \frac{x}{m\left(x^{2}+y^{2}\right)-y \sqrt{x^{2}+y^{2}}}\right),
$$

and by the parameterization $\gamma$ of the limit cycle

$$
\nabla \vartheta(\gamma(\theta))=\frac{\sqrt{m^{2}-1}}{2 \pi \beta(m-\sin (\Omega(\theta)))}(-\sqrt{\beta} \sin (\Omega(\theta)), \sqrt{\beta} \cos (\Omega(\theta))) .
$$

That is,

$$
P R C_{1}(\theta)=-\frac{\sqrt{m^{2}-1} \sin (\Omega(\theta))}{2 \pi \sqrt{\beta}(m-\sin (\Omega(\theta)))}
$$

(see Figure 2), and $P R C_{2}(\theta)=\frac{\sqrt{m^{2}-1} \cos (\Omega(\theta))}{2 \pi \sqrt{\beta}(m-\sin (\Omega(\theta)))}$.

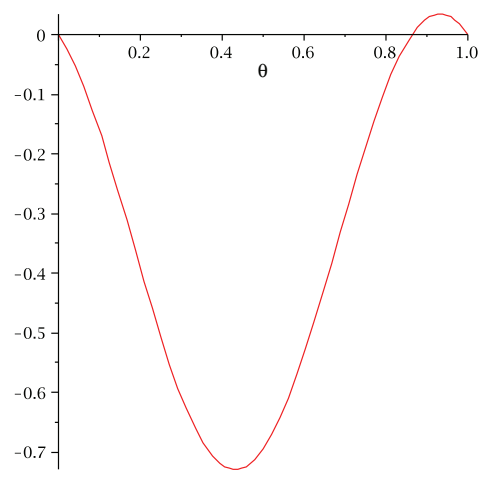

Figure 2. $P R C_{1}$ for system (5.2) with $m=1.1, \beta=1$. Observe the slightly positive bump when $\theta \in(0.9,1)$ approximately.

Copyright $($ b by SIAM. Unauthorized reproduction of this article is prohibited. 
5.1. Numerical examples. We present here an application of the method described in section 4 to a set of examples we found relevant either to illustrate the properties or because they are representative of classical models:

1. The Van der Pol oscillator:

$$
\left\{\begin{array}{l}
\dot{x}=-y+x-x^{3} \\
\dot{y}=x
\end{array}\right.
$$

2. A reduced Hodgkin-Huxley-like system, with sodium and potassium currents, and only one gating variable:

$$
\left\{\begin{array}{l}
\dot{V}=-\frac{1}{C_{m}}\left(g_{N a} m_{\infty}(V)\left(V-V_{N a}\right)+g_{K} n\left(V-V_{K}\right)+g_{L}\left(V-V_{L}\right)-I_{a p p}\right), \\
\dot{n}=n_{\infty}(V)-n,
\end{array}\right.
$$

where $V$ represents the membrane potential, $n$ is a gating variable, the open-state probability functions are

$$
m_{\infty}(V)=\frac{1}{1+\exp \left(-\left(V-V_{\max , m}\right) / k_{m}\right)}, \quad n_{\infty}(V)=\frac{1}{1+\exp \left(-\left(V-V_{\max , n}\right) / k_{n}\right)},
$$

and the parameters are $C_{m}=1, g_{N a}=20, V_{N a}=60, g_{K}=10, V_{K}=-90, g_{L}=8$, $v_{L}=-80, V_{\max , m}=-20, k_{m}=15, V_{\max , n}=-25, k_{n}=5$.

3. The Selkov model (see [26]), initially a model for self-oscillations in glycolysis, which has also been extensively used in models for circadian rhythms (see, for instance, [6] and [23]). It is given by

$$
\left\{\begin{array}{l}
\dot{x}=1-x y \\
\dot{y}=a y(x-(1+b) /(1+b y))
\end{array}\right.
$$

where the parameters are $a, b \in \mathbb{R}$.

4. The Morris-Lecar model (see [20]), initially conceived as a model for a barnacle giant muscle fiber, but well studied in the neuroscience literature (after [24]) as a paradigm for the different bifurcations that give rise to limit cycles. The model is given by

$$
\left\{\begin{aligned}
\dot{V} & =\frac{1}{C}\left(I-g_{L}\left(V-V_{L}\right)-g_{K} \omega\left(V-V_{K}\right)-g_{C a} m_{\infty}(V)\left(V-V_{C a}\right)\right), \\
\dot{w} & =\phi \frac{w_{\infty}(V)-w}{\tau_{w}(V)},
\end{aligned}\right.
$$

where

$$
\begin{aligned}
m_{\infty}(V) & =\frac{1}{2}\left(1+\tanh \left(\left(V-V_{1}\right) / V_{2}\right)\right), \\
w_{\infty}(V) & =\frac{1}{2}\left(1+\tanh \left(\left(V-V_{3}\right) / V_{4}\right)\right), \text { and } \\
\tau_{w}(V) & =\left(\cosh \left(\left(V-V_{3}\right) /\left(2 V_{4}\right)\right)\right)^{-1},
\end{aligned}
$$

and the parameters are $V_{L}=-60, V_{K}=-84, V_{C a}=120, V_{1}=-1.2, V_{2}=18, V_{3}=2$, $V_{4}=30, g_{L}=2, g_{K}=8.0, g_{C a}=4.4, C=20$, and $\phi=0.04$. These values correspond to the first case studied by Rinzel and Ermentrout in [24].

All these examples share common characteristics with slight differences that will be remarked on at the end of this section. Let us start, then, with the common features. 
5.1.1. Common features: Methods, parameter values, and figure labeling. In all the cases, we are interested in studying the dynamics close to a hyperbolic stable limit cycle $\gamma$ of period $T$ that surrounds a critical point $p^{*}$. The zero phase point on $\gamma$ is the point which has a maximum value of the first component ( $x$ or $V$ depending on the example). As in previous sections, we call $\lambda$ the characteristic exponent of $\gamma$ (so, the characteristic multiplier is $e^{\lambda}$ ). The computation of the periodic orbits has been performed using a Newton method with a tolerance of $10^{-15}$. In the neighborhood of $\gamma$, we have performed a Taylor expansion as in (4.8) up to order $L$ and we have considered $N+1$ Fourier coefficients for the $K_{n}$. With them we obtain residuals for the $K_{n}$ as defined in (4.6), which are of order $E_{\text {tail }}$. The local approximation that we get for the isochrons defined in (4.9) is computed with an error smaller than $E_{l o c}$, while the globalization of the manifold has been performed following section 4.3 and using a Taylor method with a tolerance of order $10^{-16}$. In the globalization (see definitions after (4.10)), we require a distance of order $\Delta s=10^{-2}$ between two consecutive points on the isochron, and we fix $\Delta_{\min }=10^{-8}$ and $\Delta \alpha=0.3$.

Values for each example of all the parameters defined in the last paragraph are given in Table 1. All the results will be given with four significant digits, although all the computations have been performed with double precision.

Table 1

Parameter values for the different models: $T=$ period of the orbit $\gamma ; \lambda=$ characteristic exponent associated to $\gamma ; L=$ order of the Taylor expansion; $N+1=$ number of Fourier coefficients; $E^{\text {tail }}=$ residuals for the $K_{n}$; $E_{l o c}=$ maximum error when computing local approximation of isochrons.

\begin{tabular}{|c|c|c|c|c|c|c|c|}
\hline Model & Figure & $T \approx$ & $\lambda \approx$ & $L=$ & $N=$ & $E_{\text {tail }} \in$ & $E_{\text {loc }}=$ \\
\hline 1 & 3 & 6.663 & -7.059 & 15 & $2^{8}=256$ & $\left(10^{-20}, 10^{-15}\right)$ & $10^{-12}$ \\
\hline $2, I_{\text {app }}=10$ & 4 & 7.074 & -27.66 & 5 & $2^{11}=2048$ & $\left(10^{-17}, 10^{-12}\right)$ & $10^{-8}$ \\
\hline $2, I_{\text {app }}=165$ & 5 & 1.630 & -3.384 & 10 & $2^{9}=512$ & $\left(10^{-19}, 10^{-14}\right)$ & $10^{-10}$ \\
\hline 3 & 6 & 6.344 & -4.909 & 15 & $2^{9}=512$ & $\left(10^{-16}, 10^{-14}\right)$ & $10^{-11}$ \\
\hline 4 & 7 & 99.27 & -9.122 & 5 & $2^{10}=1024$ & $\left(10^{-20}, 10^{-13}\right)$ & $10^{-8}$ \\
\hline
\end{tabular}

For each model, we present a figure (Figures 3 to 7 ) with different panels. In order to compact notation, we label each panel with a different symbol: Kn, Iso, PRC, PRS, and PR $\theta$.

In panels $\mathrm{Kn}$, the computed $K_{n}$ for some values of $n$ are shown. The fact that the orbits do not approach $\gamma$ uniformly in $\theta$ has the effect that for certain values of $\theta$, as $n$ goes to infinity, the value of $K_{n}$ is not uniform in $\theta$; see the slow-fast item in section 6 for a discussion on this question.

In panels Iso we plot the isochrons corresponding to the phases $j / N_{\theta}$ for $j=0, \ldots, N_{\theta}-1$, with $N_{\theta}=16$, typically. We show the local approximation (black) computed semianalytically using the parameterization method and the globalized isochron (red) using the dynamics given by the vector field. We restrict the computation to a rectangular domain $\mathcal{R}$ in the variables $(x, y)$ containing the limit cycle.

In panels $\mathrm{PRC}$ we plot the $\mathrm{PRC}_{1}$ (red) and the $\mathrm{PRC}_{2}$ (blue) for an infinitesimally small perturbation in the directions $(1,0)$ and $(0,1)$, respectively, with a certain amplitude specified in each figure caption, jointly with the $x$ (or $V$ ) component (dashed line) of the oscillator (scaled for a better reference). Notice that the $\mathrm{PRC}_{1}$, corresponding to the horizontal pulses,

Copyright (c) by SIAM. Unauthorized reproduction of this article is prohibited. 
is just the section with $\sigma=0$ of the PRSs that are given in panels PRS.

In panels PRS, we plot the $\mathrm{PRS}_{1}$ in the variables $\theta$ and $\sigma$, but only for positive values of $\sigma$, avoiding negative values for the sake of clarity. The positive values of $\sigma$ correspond to the points $(x, y)$ in the external part of the limit cycle (depending on the orientation of the limit cycle, the sign of $\sigma$ defined by the parameterization method out of the limit cycle can be also negative; in these figures we change $\sigma \mapsto-\sigma$ for the sake of homogeneity). Indeed, in these panels we plot the phase shift for the points $(x, y)$ in the outer neighborhood of the limit cycle displayed in panels Iso. Since we restrict our attention to the rectangular domain $\mathcal{R}$ in the variables $(x, y)$ (because they are the "real" phase space variables), when plotting the PRS in the variables $(\theta, \sigma)$, we come across a nonregular domain. Additionally, sometimes the discretization of local isochrons $(\Delta s)$ used to globalize other isochrons undergoes the limit $\Delta_{\min }$ and can no longer extend the isochron. This is why some isochrons do not reach the border of the rectangular domain $\mathcal{R}$.

Like the isochrons, the $\mathrm{PRS}_{1} \mathrm{~s}$ are computed locally using semianalytical methods and extended by integrating the system (3.11); see section 4.3.

Although the PRSs contain the maximum information about phase advancement, sometimes they are not easy to visualize. Accordingly, we have decided to show sections of the PRSs with fixed phases (that is, $\theta=\theta^{*}$ and parameterized by $\sigma$ ). Using this view, we can easily illustrate the differences in phase advancement between different points on the same isochron. These panels are labeled as $\operatorname{PR} \theta$.

Before the discussion, we go through the noncommon features of each example. In the following list M stands for Model.

M2. For the Hodgkin-Huxley-like model (5.5), we have studied the system in two regimes: $I_{a p p}=10$ (close to a SNIC bifurcation which occurs at $I_{a p p} \approx 4.513$ ) and $I_{a p p}=165$ (close to a Hopf bifurcation which occurs at $I_{a p p} \approx 213.8$ ). Bifurcation values are obtained through XPPAUT; see [7].

For the case $I_{a p p}=10$, which is the case close to SNIC, the system presents a slow-fast dynamics that will accentuate some of the problems that we already mentioned in the previous example. In this case, we computed the Taylor expansion up to order $L=5$, because, as we can appreciate in Figure 4, for some values of $\theta$, as $n$ increases the $K_{n}$ tend to zero much faster. Moreover, since the functions $K_{n}$ present very sharp spikes we need to consider up to $2^{10}=1024$ or $2^{11}=2048$ Fourier modes to get good approximations, that is, with residuals smaller than a certain error. This implies solving linear systems with large matrices that are not very stable.

Notice that this limit cycle is "strongly" hyperbolic and the backward integration can be somehow very unstable.

The system presents a fixed point at $(-26.83,0.4093)$, which is computed using a Newton method with a tolerance of $10^{-13}$.

One can appreciate in Figure 4 that the isochrons computed semianalytically (the green ones, which are hardly noticeable) are shorter than in the Hopf case (Figure 5). In this case, in order to have a long enough local approximation for the isochrons, we reduced the accuracy of the computation down to $10^{-8}$.

For the case $I_{a p p}=165$, which is close to the Hopf bifurcation, in Figure 5 one can observe that the slow-fast phenomenon that we mentioned above is softened. The system presents a

Copyright (c) by SIAM. Unauthorized reproduction of this article is prohibited. 
hyperbolic fixed point at $(-21.00,0.6899)$, which has been computed numerically with an error smaller than $10^{-13}$.

M3. The Selkov model (5.6) presents an Andronov-Hopf bifurcation for $a=(1+b) / b$. We have considered here the case $a=3, b=1$. The unstable fixed point, which can be easily computed analytically, is located at $(1,1)$.

In this example, we have decided to make the starting points on the isochron closer to one another, because points diverge very fast far from the limit cycle; consequently, we allow $\Delta_{\min }=10^{-12}$.

M4. For the Morris-Lecar model (5.7), we have considered the case $I_{a p p}=91$, which presents issues similar to the two-dimensional Hodgkin-Huxley model close to a SNIC considered above.

The system has a fixed point at $(-26.26,0.1320)$, which is computed using a Newton method with a tolerance of $10^{-13}$.

It is to be noticed that (5.7) presents a subcritical Hopf bifurcation at $I_{a p p} \approx 93.86$; the unstable limit cycle goes "back" in the parameter space up to $I_{a p p} \approx 88.29$, where it coalesces with a stable limit cycle in a bifurcation of a semistable limit cycle that disappears for lower $I_{a p p}$ 's. The stable limit cycle, which comes from another bifurcation for some $I_{a p p} \gg 93.86$, is the one that we study. It can be checked that the period of this stable orbit is notably above of that of the unstable orbit, born at the subcritical Hopf bifurcation; thus, one may expect an accentuated slow-fast dynamics, more similar to a limit cycle close to a SNIC bifurcation than to a Hopf bifurcation.

Some of the shortcomings of the Hodgkin-Huxley model close to a SNIC are also reproduced here: it is necessary to solve linear systems with large matrices that are not very stable; the limit cycle is "strongly" hyperbolic and the backward integration can be somehow very unstable; and, finally, to have a long enough local approximation for the isochrons, we need to reduce the accuracy of the computation.

Another specific observation is that the isochrons spiral around the unstable limit cycle in the interior of the stable one. This is not surprising since the two limit cycles have different periods, and so they cannot share the system of isochrons. The next short example analytically illustrates this fact.

Example 5.3. Consider the $\mathcal{C}^{1}$ system in polar coordinates

$$
X:=\left\{\begin{array}{l}
\dot{r}=r a(r) \\
\dot{\theta}=b(r)
\end{array}\right.
$$

Suppose that $a\left(r_{j}\right)=0, a^{\prime}\left(r_{j}\right) \neq 0$, and $b\left(r_{j}\right) \neq 0$ for $j=1,2$, with $r_{1} \neq r_{2}$, both positive.

It is straightforward to see that $\left\{r=r_{1}\right\}$ and $\left\{r=r_{2}\right\}$ are hyperbolic limit cycles of $X$ with alternate stability. From [10], we can deduce that, for each limit cycle, the vector field

$$
Y_{j}:=\left\{\begin{array}{l}
\dot{r}=r \\
\dot{\theta}=\left(b(r)-b\left(r_{j}\right)\right) /(a(r))
\end{array}\right.
$$

satisfies $\left[Y_{j}, X\right]=\mu Y_{j}$, with $\mu(r)=r a^{\prime}(r)$.

Now let us take $Y_{1}$, for instance. It is clear that, in general, $\dot{\theta}$ is not defined on $r=r_{2}$ since $a\left(r_{2}\right)=0$. However, if $b\left(r_{2}\right)=b\left(r_{1}\right)$ (both limit cycles have the same period!), then $\dot{\theta}$ 

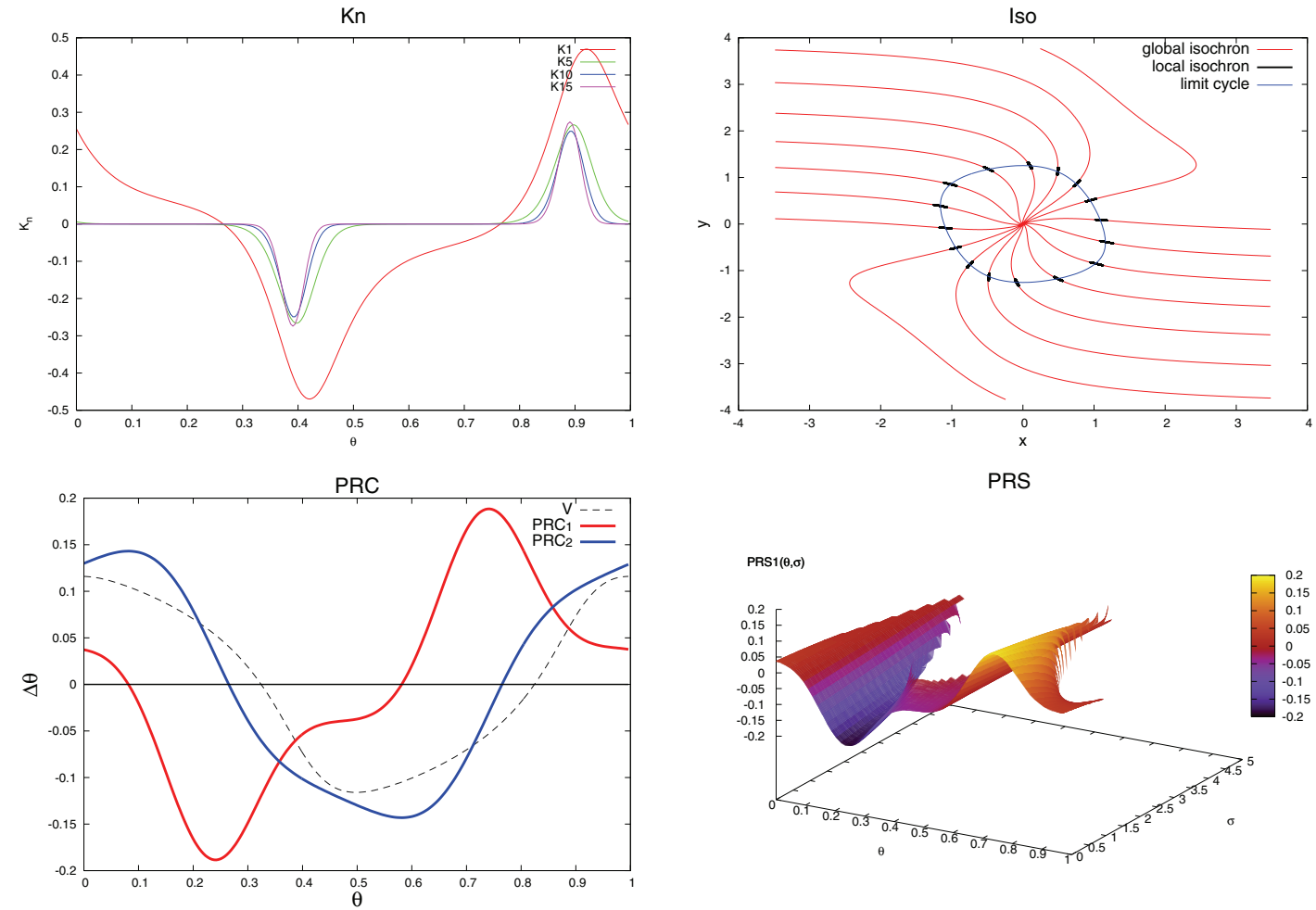

PRS

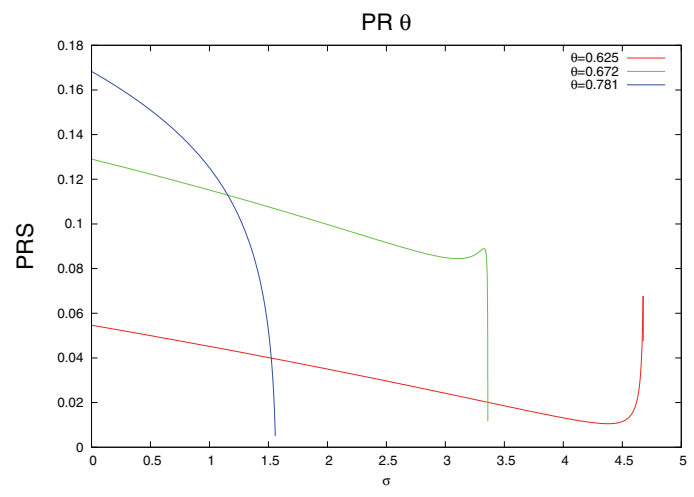

Figure 3. The van der Pol oscillator. In the panel PRC, we show $P R C_{1}$ and $P R C_{2}$ with amplitude 1 and $x$ scaled by a factor 0.1. See section 5.1.1 for a general explanation about the contents of each panel. In panel $\operatorname{PR} \theta$, notice the diversity of phase advancements that can be obtained in the same isochron (three isochrons are shown: $\theta=0.625,0.672,0.781)$.

may be extended on $r=r_{2}$, and thus $\left\{r=r_{2}\right\}$ may be contained in the domain $\Omega$, where the isochrons of $\left\{r=r_{1}\right\}$ are defined. This is the case, for instance, when the system is rigid $(\dot{\theta}=$ constant $)$, for which the isochrons are straight lines from the origin.

In the case that $b\left(r_{2}\right) \neq b\left(r_{1}\right)$ (different periods like the numerical example illustrated in Figure 7), $\dot{\theta}$ in $Y_{1}$ is not bounded close to $\left\{r=r_{2}\right\}$, and, then, the isochrons of $\left\{r=r_{1}\right\}$ spiral around.

Copyright (c) by SIAM. Unauthorized reproduction of this article is prohibited. 

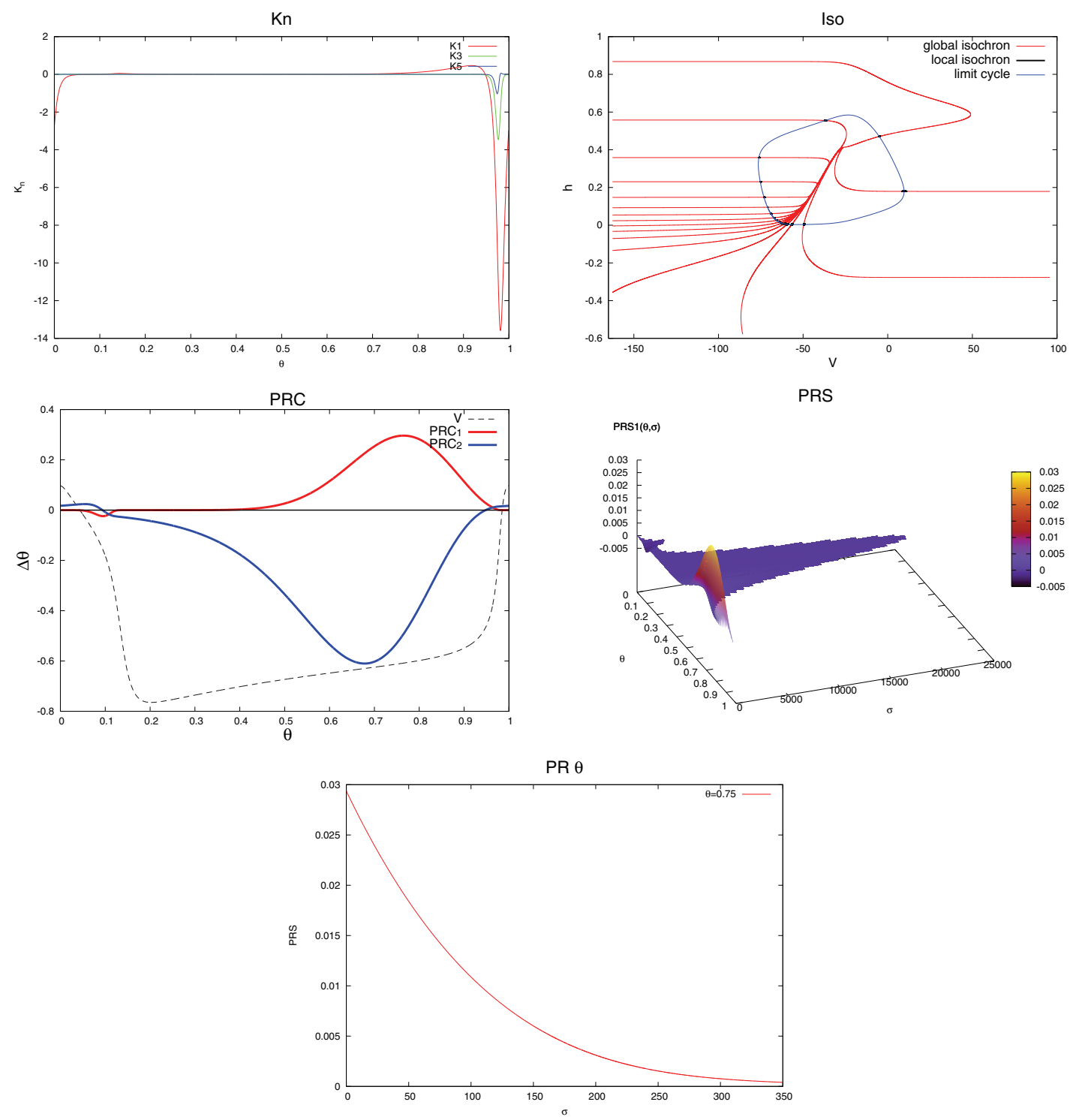

Figure 4. Two-dimensional Hodgkin-Huxley close to a SNIC bifurcation. In the panel PRC, we show PRC 1 and $\mathrm{PRC}_{2}$ with amplitude 10 and 0.1 , respectively, and $V$ scaled by a factor 0.01 . See section 5.1 .1 for a general explanation about the contents of each panel. In panel Iso, notice the heterogeneity in the distances between isochrons with equidistant phases, thus reflecting the slow-fast nature of the system.

6. Discussion. Generally, we want to emphasize the different approaches used in this paper. The main goal is to compute a biologically interesting object, the PRSs, but it involves the computation of isochrons in a rigorous way. To achieve this intermediate purpose, we have related different ways of understanding the dynamics in a neighborhood of an isochronous limit cycle: the geometrical approach given by the Lie symmetries and analytical methods to compute invariant manifolds. Both have been useful either to express or to compute the PRSs.

Copyright $\odot$ by SIAM. Unauthorized reproduction of this article is prohibited. 

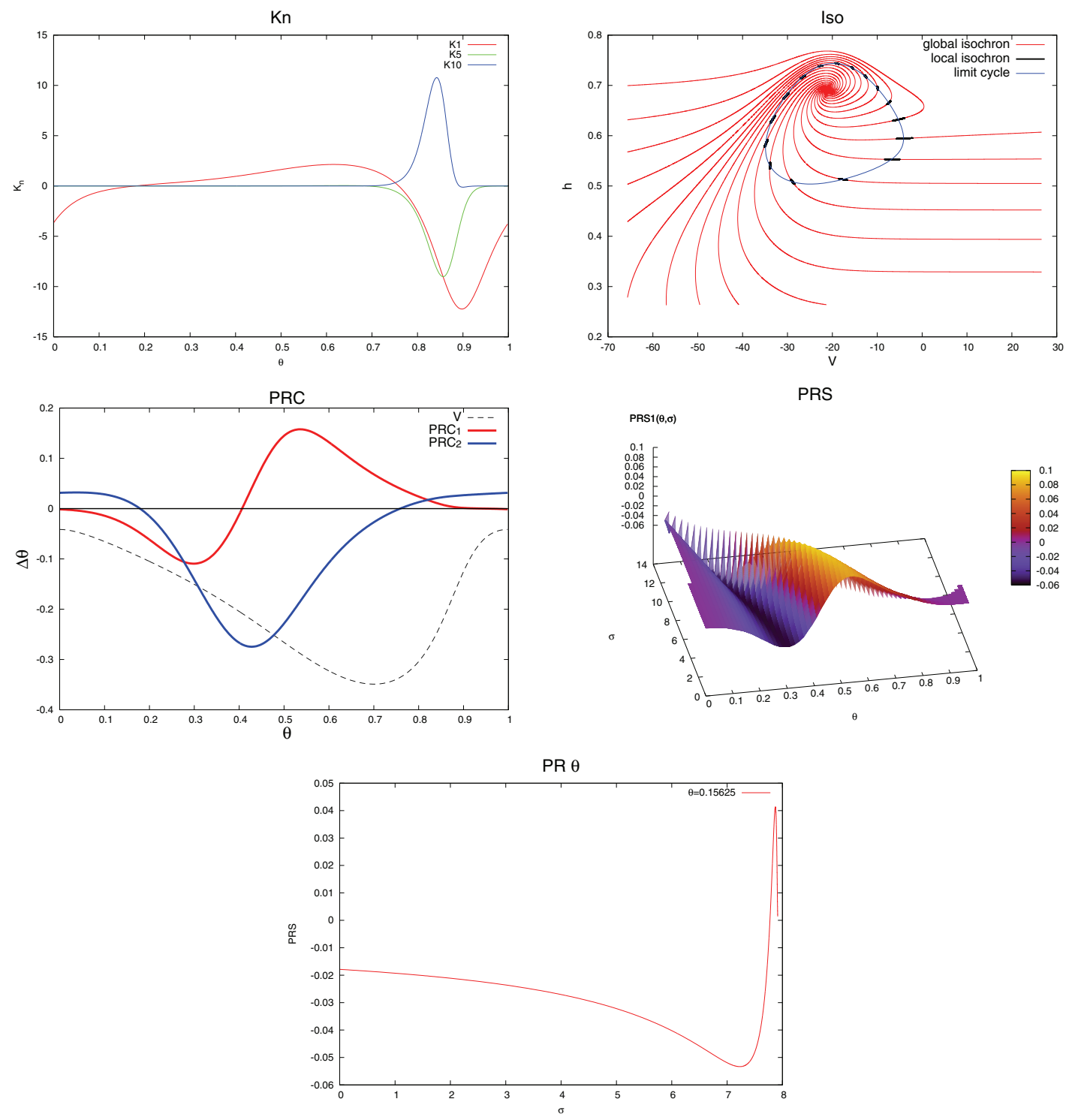

Figure 5. Two-dimensional Hodgkin-Huxley close to a Hopf bifurcation. In the panel PRC, we show PRC 1 and $\mathrm{PRC}_{2}$ with amplitude 2 and 0.02 , respectively, and $V$ scaled by a factor 0.01 . See section 5.1 .1 for a general explanation about the contents of each panel.

We have also paid careful attention to the numerical aspects involved in the method, which are not trivial, and showed relationships among the geometry of the orbits, the dynamics of the vector field, and the numerical schemes. Concerning the practical part of the effective computation, the above examples have shed light upon different biological and numerical issues that we would like to discuss next.

From “Type 1" to "Type 2" PRCs. As we mentioned in the introduction, from [8], PRCs are classified as models with strictly positive or mainly positive PRCs ("Type 1" or "Class 1") 

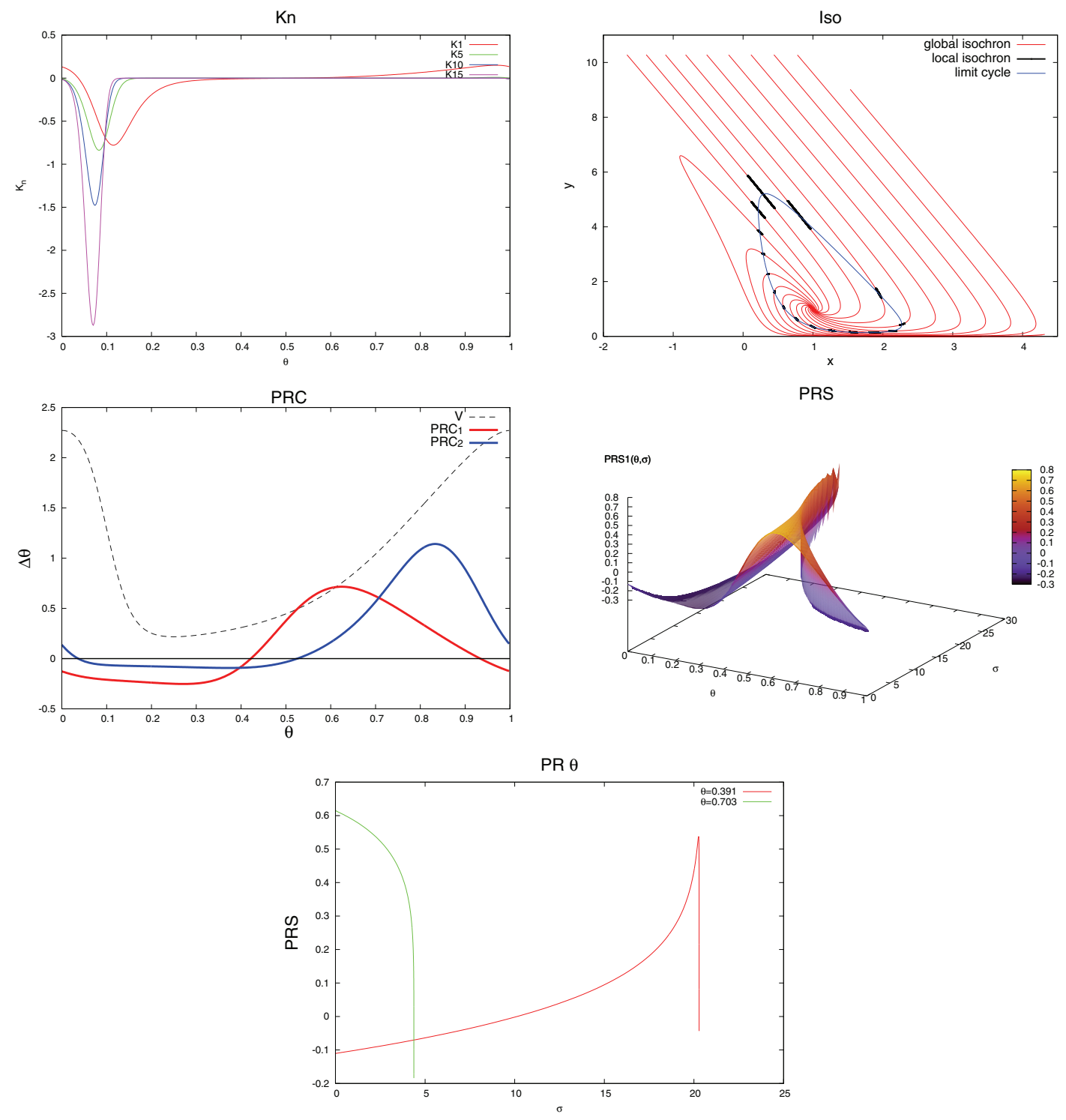

Figure 6. Selkov model with $a=3$ and $b=1$, not far from a Hopf bifurcation. See section 5.1 .1 for a general explanation about the contents of each panel.

and models whose PRCs change sign ("Type 2" or "Class 2"). The rule of thumb proposed by Ermentrout is that Type 1 PRCs correspond to models in which oscillations appear via saddle-node on invariant circle bifurcations, whereas Type 2 PRCs correspond to supercritical Andronov-Hopf bifurcations.

Our examples confirm this rough classification, and we have used them to give an idea of how the transition between the two PRC types takes place. Another study, using only PRCs and continuation methods, has recently appeared in [11].

Analytically, we have seen, for instance (see Example 5.1), that $P R C_{1}(\theta)=$ $-\sin (2 \pi \theta) /(2 \pi \sqrt{\beta})$ close to a Hopf bifurcation and (see Example 5.2) $P R C_{1}(\theta)=$ 

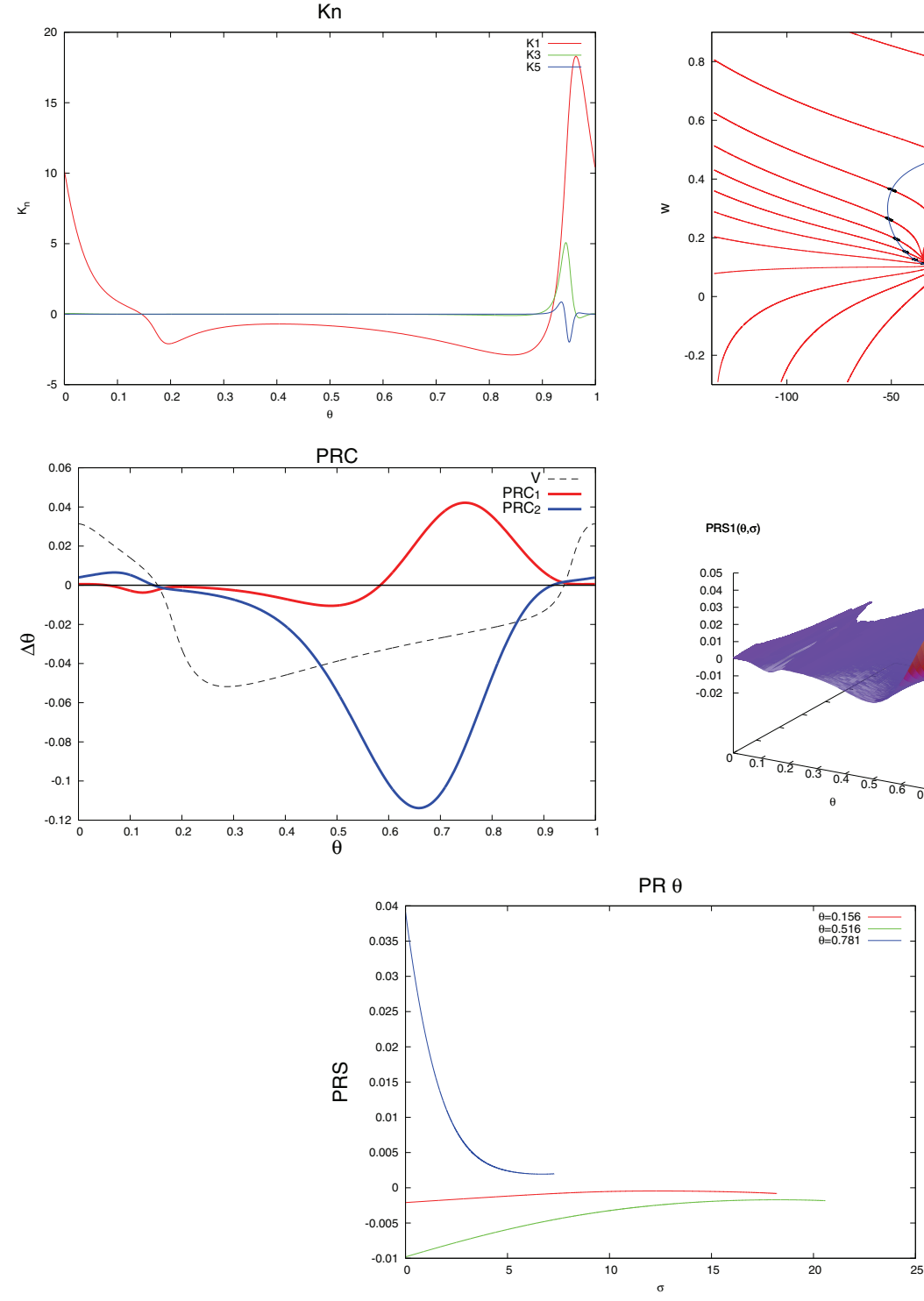

Figure 7. Morris-Lecar model. In the panel PRC, we show $P R C_{1}$ and $P R C_{2}$ with amplitude 1 and 0.01 , respectively, and $V$ scaled by a factor 0.001. See section 5.1.1 for a general explanation about the contents of each panel. In panel Iso, notice how isochrons spiral around the unstable limit cycle; see also the paragraph before Example 5.3, on page 1031.

$-\sqrt{m^{2}-1} \sin (\Omega(\theta)) /(2 \pi \sqrt{\beta}(m-\sin (\Omega(\theta)))$, where $\Omega$ is given in (5.3), for a system that presents a SNIC bifurcation at $m=1$ (see also Figure 2).

These examples clearly show that the fact that systems with oscillations coming from a saddle-node bifurcation are of "Type 1," that is, the PRC is mainly positive (or mainly negative), is produced by the slow-fast dynamics; moreover, the time it spends on a negative (resp., positive) regime is very short compared to the time it spends out of it. This fact can also be observed when comparing (5.5) with $I_{a p p}=10$ (see Figure 4) or (5.5) with $I_{a p p}=165$

Copyright ( $\odot$ by SIAM. Unauthorized reproduction of this article is prohibited. 
(see Figure 5). For instance, in Figure 4, panel PRC, we can appreciate a small negative portion of the $\mathrm{PRC}_{1}$ (close to $\theta=0.1$ ), whereas in the same panel of Figure 5 , the negative part has a bigger area. As the value $I_{a p p}$ increases from $I_{a p p}=10$, the negative portion of the PRC is enlarging up to the bifurcation point $I_{a p p} \approx 213.8$, where the PRC has practically zero integral.

Observe also that systems with a marked "Type II" tendency (mainly, (5.4), (5.5) with $I_{a p p}=165$, or (5.6)) reach the extreme values of the PRCs where the isochrons have maximal curvature nearby the limit cycle (the correspondence is not exact because it also depends on the stimulus direction). Compare panels PRC with panels Iso in Figures 3, 5, and 6. On the other hand, the chosen value for the Morris-Lecar system (see Figure 7) shows an intermediate behavior, perhaps closer to "Type II" than to "Type I" (recall from M4 that it takes place close to a double limit cycle bifurcation).

Numerical drawbacks for slow-fast systems. From a joint analysis of panels Kn and Iso in Figures 3 to 7, we can deduce the effect of slow versus fast dynamics.

The fact that we impose the same dynamics $(2.7)$ in the variables $(\theta, \sigma)$ in the whole neighborhood of the limit cycle has a dramatic effect when the system presents two different dynamics (slow and fast).

On the one hand, the slow-fast dynamics causes the presence of spikes in the functions $K_{n}$, and when trying to approximate this type of function using Fourier series one faces the problem that the series converge slowly and not uniformly, and therefore one needs to keep a large number of harmonics in order to approximate $K_{n}$. This phenomenon is clearly observed in system (5.5) close to the SNIC bifurcation (see also the panel Kn in Figure 4).

Moreover, the presence of two types of dynamics can be thought of as if the periodic orbit possesses different rates of contraction (in different places) which are remarkably different. When imposing a uniform $\lambda$ in a neighborhood of the limit cycle $\gamma$, the change of coordinates $K$ that conjugates the dynamics to (3.6) is not uniform in $\theta \in \mathbb{T}$. That is, for some values of $\theta \in \mathbb{T}$, the functions $K_{n}$ take values with orders of magnitude above the rest of the cycle; in other terms, $\frac{\max _{\theta}\left|K_{n}(\theta)\right|}{\min _{\theta}\left|K_{n}(\theta)\right|} \gg 1$. The numerical problem is that, in this case, it is impossible to choose a uniform $b$ (see Remark 4.5) such that the $K_{n}$ can consistently have order 1 for all the values of $\theta$. Thus, for these values of $\theta$, increasing the degree $L$ of the Taylor polynomial does not have any effect on the growth of the domain where the isochron can be computed semianalytically. This situation turns out to be a serious issue for the cases when the systems present an accentuated slow-fast dynamics (see panel Kn in Figure 4).

Role of PRSs under high frequency stimulations. As explained in section 1, the phase advancement computed on the limit cycle (PRC) can differ from that computed out of the limit cycle (PRS). This difference will be important under different circumstances like a short period of stimulation, a slow attraction to the limit cycle, a large stimulus amplitude, environmental random fluctuations, and bursting-like stimuli. We now fix our attention to panels PRS and PR $\theta$ in all the figures. Our purpose is to highlight the differences in the phase advancement for points in a neighborhood of $\gamma$ which share the same phase $\theta$.

As with the maximum and minimum values of the PRCs, we pay attention to the zones close to the limit cycle where the curvature of the isochrons is extreme. This phenomenon can be timidly observed in Figure 4, panel $\operatorname{PR} \theta$, where the section $\theta=0.75$ of the PRS is

Copyright (c) by SIAM. Unauthorized reproduction of this article is prohibited. 
shown to be decreasing. More exaggerated variations can be obtained for $\theta=0.15625$ in Figure 5, panel PR $\theta$, or in Figure 3. In the latter case, we show a $z o o$ of possible sections $(\theta \in\{0.672,0.625,0.781\})$.

Changes of the phase advancement with respect to $\sigma$ given a fixed $\theta$, as the above examples show, combined with high frequency stimuli, rule out the possibility of controlling the whole phase advancement of an experiment using only the PRCs. Thus, the PRSs become extremely useful. In our examples, these differences are more noticeable close to Type II oscillators (Hopf) because of the stronger curvature of the isochrons.

Our results agree with the fact that perturbations applied to Type II oscillators produce significant normal displacements from the limit cycle rather than those applied to Type I. This fact has also been studied in [22] by Oprisan and Canavier, in the sense that the difference in angular velocity at displaced points compared to the angular velocity on the limit cycle is then more important. As pointed out in [22], this might affect the study of biological circuits comprising Type II neural oscillators, which appear frequently in identified central pattern-generating circuits.

Isochrons in higher dimensions. Although in this paper we apply the method only to compute isochrons and PRSs to planar differential systems, it can be applied to higher dimensions provided that the limit cycle is isochronous (for instance, if it is hyperbolic and stable). The formulation in terms of Lie symmetries, the parameterization method, and their mutual relationship will still be valid to describe the isochronous sections in higher dimensions.

On one hand, it is not difficult to see that a limit cycle $\gamma$ of a vector field $X \in \mathbb{R}^{n}, n \geq 2$, is isochronous if there exist $n-1$ nontrivial vector fields $Y_{1}, \ldots, Y_{n-1}$ in involution (see [21]) satisfying the Lie symmetry equation (2.4).

On the other hand, for stable isochronous limit cycles, the parameterization method would allow us to compute the isochrons which correspond, in this case, to $(n-1)$-dimensional hypersurfaces transversal to $\gamma$.

Appendix A. Proof of Theorem 3.1. In order to prove Theorem 3.1, we will see that $(1) \Leftrightarrow(2)$ and $(2) \Leftrightarrow(3)$. Notice first that $(1) \Leftrightarrow(2)$ was already proved in Theorem 4 in [25]. Let us prove $(2) \Leftrightarrow(3)$, and we will see that $(2) \Rightarrow(3)$.

Assume there exist a vector field $Y$ and a scalar function $\mu$ satisfying the Lie symmetry (2.4), and consider $K(\theta, \sigma)=\psi_{\sigma}(\gamma(\theta))$, where $\psi_{\sigma}$ is the flow associated to the vector field $Y$ and $\gamma(\theta)$ is the parameterization of the periodic orbit of the vector field $X$.

Then, notice that

$$
\partial_{\sigma}(X(K(\theta, \sigma)))=D X(K(\theta, \sigma)) \partial_{\sigma} K(\theta, \sigma)=D X(K(\theta, \sigma)) Y(K(\theta, \sigma)) .
$$

Using the Lie symmetry $D X Y-D Y X=\mu Y$, we have that

$$
\partial_{\sigma}(X(K(\theta, \sigma)))=D Y(K(\theta, \sigma)) X(K(\theta, \sigma))+\mu(K(\theta, \sigma)) Y(K(\theta, \sigma)) .
$$

Hence, $X(K(\theta, \cdot))$ is a solution of the linear equation

$$
\frac{d}{d \sigma} Q=(D Y \circ K)(\theta, \sigma) Q+(\mu Y \circ K)(\theta, \sigma)
$$

Copyright $\odot$ by SIAM. Unauthorized reproduction of this article is prohibited. 
with the initial condition

$$
Q(0)=X(K(\theta, 0))=X(\gamma(\theta))
$$

Let $\Psi_{\sigma}$ be the fundamental solution of the homogeneous equation

$$
\frac{d}{d \sigma} Q=(D Y \circ K)(\theta, \sigma) Q
$$

then, the variation of parameters formula tells us that the solution of (A.1) with initial condition (A.2) is given by

$$
Q_{\theta}(\sigma)=\Psi_{\sigma} X(\gamma(\theta))+\Psi_{\sigma} \int_{0}^{\sigma} \Psi_{s}^{-1} \mu(K(\theta, s)) Y(K(\theta, s)) d s .
$$

Notice that $\Psi_{s}^{-1} Y(K(\theta, s))$ is independent of $s$, since

$$
\begin{aligned}
\partial_{s}\left(\Psi_{s}^{-1} Y(K(\theta, s))\right)= & -\Psi_{s}^{-1} D Y(K(\theta, s)) \Psi_{s} \Psi_{s}^{-1} Y(K(\theta, s)) \\
& +\Psi_{s}^{-1} D Y(K(\theta, s)) Y(K(\theta, s)) \\
= & 0
\end{aligned}
$$

for all $s$. Then we can take $\Psi_{s}^{-1} Y(K(\theta, s))=\Psi_{\sigma}^{-1} Y(K(\theta, \sigma))$, and we are left with the following expression for $X \circ K$ :

$$
X(K(\theta, \sigma))=\Psi_{\sigma} X(\gamma(\theta))+\Psi_{\sigma} \Psi_{\sigma}^{-1} Y(K(\theta, \sigma)) \int_{0}^{\sigma} \mu(K(\theta, s)) d s .
$$

Finally, using that the parameterization $K$ was defined by the orbits of the vector field $Y$ for the points on the limit cycle $\gamma$ (see (3.3)), we have

$$
\partial_{\theta} K(\theta, \sigma)=\partial_{\theta} \psi_{\sigma}(\gamma(\theta))=D \psi_{\sigma}(\gamma(\theta)) T X(\gamma(\theta))=T \Psi_{\sigma} X(\gamma(\theta))
$$

and the expression for $X(K(\theta, \sigma))$ reads as

$$
X(K(\theta, \sigma))=\left(\frac{1}{T} \partial_{\theta}+\left(\int_{0}^{\sigma} \mu(K(\theta, \tau)) d \tau\right) \partial_{\sigma}\right) K(\theta, \sigma) .
$$

Therefore, taking

$$
F(\theta, \sigma)=\int_{0}^{\sigma} \mu(K(\theta, \tau)) d \tau
$$

we obtain the claimed result.

The other implication follows in the following way. Let $K$ be a smooth map satisfying (3.1). Consider $Y$ the vector field whose orbits for the points on the limit cycle $\gamma(\theta)$ are given by $\{K(\theta, \sigma) \mid \sigma \in \mathbb{R}\}$. Let $\sigma$ be the integration time along the orbits of the vector field $Y$. Then,

$$
Y(K(\theta, \sigma))=\partial_{\sigma} K(\theta, \sigma)
$$


The fact that the curves $\left\{K\left(\theta_{0}, \sigma\right) \mid \sigma \in \mathbb{R}\right\}$ are transversal to the orbits of $X$ implies also that $Y$ is transversal to $X$.

We next prove that $X$ is a normalizer of the vector field $Y$. From (3.1), taking derivatives with respect to $\sigma$, we get

$$
\left(\frac{1}{T} \partial_{\theta}+F(\theta, \sigma) \partial_{\sigma}\right) \partial_{\sigma} K+\partial_{\sigma} F \partial_{\sigma} K=(D X \circ K) \partial_{\sigma} K
$$

and using (A.4), we get

$$
\left(\frac{1}{T} \partial_{\theta}+F(\theta, \sigma) \partial_{\sigma}\right)(Y \circ K)+\partial_{\sigma} F(Y \circ K)=(D X \circ K)(Y \circ K) .
$$

By the chain rule,

$$
(D Y \circ K)\left(\frac{1}{T} \partial_{\theta}+F(\theta, \sigma) \partial_{\sigma}\right) K+\partial_{\sigma} F(Y \circ K)=(D X \circ K)(Y \circ K),
$$

and again, by the invariance equation (3.1), we obtain

$$
(D X \circ K)(Y \circ K)-(D Y \circ K)(X \circ K)=\partial_{\sigma} F(Y \circ K),
$$

and taking $\mu \circ K=\partial_{\sigma} F$, we have $[Y, X]=\mu Y$, as we wanted to prove.

Appendix B. Proof of Proposition 3.6. Let us prove that if one considers the function $\nabla \vartheta$ along the orbits $\phi_{t}(p)$ of $X$, it satisfies the adjoint equation (3.11). Recall that, by (3.8), we have that

$$
\nabla \vartheta\left(\phi_{t}(p)\right)=\frac{Y^{\perp}\left(\phi_{t}(p)\right)}{T\left\langle Y^{\perp}\left(\phi_{t}(p)\right), X\left(\phi_{t}(p)\right)\right\rangle}
$$

where $Y$ satisfies (2.4) for some $\mu$. In order to check this statement, we first introduce the matrix $J$ given by

$$
J=\left(\begin{array}{cc}
0 & -1 \\
1 & 0
\end{array}\right)
$$

such that $Y^{\perp}=J Y$. Notice that for a $2 \times 2$ real matrix $A$ we have

$$
(J A)-(J A)^{T}=\operatorname{tr}(A) J
$$

where $\operatorname{tr}(A)$ denotes the trace of the matrix $A$.

Now, we consider the derivative of $\nabla \vartheta\left(\phi_{t}(p)\right)$ with respect to the time. In order to simplify notation we set $x:=\phi_{t}(p)$ and $g(x):=\left\langle Y^{\perp}(x), X(x)\right\rangle$. Using that $\frac{d}{d t} Y(x)=D Y(x) X(x)$, we have from (B.1)

$$
\frac{d}{d t} \nabla \vartheta(x)=\frac{J D Y(x) X(x)}{T g(x)}-\frac{Y^{\perp}(x)(\langle J D Y(x) X(x), X(x)\rangle+\langle J Y(x), D X(x) X(x)\rangle)}{T g(x)^{2}} .
$$

Copyright $\odot$ by SIAM. Unauthorized reproduction of this article is prohibited. 
Using now the Lie symmetry $D X Y-D Y X=\mu Y$, expression (B.1), and dot product properties (namely, $\left.\langle J Y(x), D X(x) X(x)\rangle=\left\langle D X(x)^{T} J Y(x), X(x)\right\rangle\right)$, we obtain

$$
\begin{aligned}
\frac{d}{d t} \nabla \vartheta(x) & =\frac{J D X(x) Y(x)-\mu(x) J Y(x)}{T g(x)} \\
& -\frac{\nabla \vartheta(x)\left(\left\langle J D X(x) Y(x)-\mu(x) J Y(x)+D X(x)^{T} J Y(x), X(x)\right\rangle\right)}{g(x)} .
\end{aligned}
$$

Applying (B.3) and $(J D X(x))^{T}=-D X(x)^{T} J$ and denoting $\tau(x):=\operatorname{tr}(D X)(x)$, we are led to

$$
\frac{d}{d t} \nabla \vartheta(x)=\frac{\left(-D X(x)^{T}+\tau(x)-\mu(x)\right) J Y(x)}{T g(x)}-\frac{\nabla \vartheta(x)(\langle(\tau(x)-\mu(x)) J Y(x), X(x)\rangle)}{g(x)} .
$$

In fact, again since $\nabla \vartheta(x)=(J Y(x)) /(T g(x))$ and $\langle J Y, X\rangle / g(x)=1$, we have

$$
\frac{d}{d t} \nabla \vartheta(x)=\left(-D X(x)^{T}+\tau(x)-\mu(x)\right) \nabla \vartheta(x)-\nabla \vartheta(x)(\tau(x)-\mu(x))=-D X(x)^{T} \nabla \vartheta(x),
$$

as we wanted to prove.

Acknowledgments. The authors want to especially thank Rafael de la Llave for his guidance in the use of the parameterization methods. We are also very grateful to R. Calleja, A. Delshams, A. Jorba, A. Luque, P. Roldán, J. Villanueva, and M. Zou, with whom we have had fruitful discussions.

\section{REFERENCES}

[1] E. O. Brigham, The Fast Fourier Transform and Its Applications, Prentice-Hall, Englewood Cliffs, NJ, 1988.

[2] E. Brown, P. Holmes, And J. Moehlis, On the phase reduction and response dynamics of neural oscillator populations, Neural Comp., 16 (2004), pp. 673-715.

[3] X. Cabré, E. Fontich, and R. DE la Llave, The parameterization method for invariant manifolds. III. Overview and applications, J. Differential Equations, 218 (2005), pp. 444-515.

[4] C. Cajochen, K. Kräuchi, And A. Wirz-Justice, Role of melatonin in the regulation of human circadian rhythms and sleep, J. Neuroendocrinology, 15 (2003), pp. 432-437.

[5] C. Chicone And W. Liu, Asymptotic phase revisited, J. Differential Equations, 204 (2004), pp. $227-246$.

[6] A. Diez-Noguera, A functional model of the circadian system based on the degree of intercommunication in a complex system, Am. J. Physiol. Regul. Integr. Comp. Physiol., 267 (1994), pp. R1118-R1135.

[7] B. Ermentrout, Simulating, Analyzing, and Animating Dynamical Systems: A Guide to XPPAUT for Researchers and Students, Software Environ. Tools 14, SIAM, Philadelphia, 2002.

[8] G. B. Ermentrout, Type I membranes, phase resetting curves, and synchrony, Neural Comp., 8 (1996), pp. $979-1001$.

[9] G. B. Ermentrout And N. Kopell, Multiple pulse interactions and averaging in systems of coupled neural oscillators, J. Math. Biol., 29 (1991), pp. 195-217.

[10] E. Freire, A. Gasull, And A. Guillamon, Limit cycles and Lie symmetries, Bull. Sci. Math., 131 (2007), pp. 501-517.

[11] W. GovaerTs And B. Sautois, Computation of the phase response curve: A direct numerical approach, Neural Comp., 18 (2006), pp. 817-847.

[12] A. Griewank, Evaluating Derivatives: Principles and Techniques of Algorithmic Differentiation, Frontiers Appl. Math. 19, SIAM, Philadelphia, 2000.

Copyright (C) by SIAM. Unauthorized reproduction of this article is prohibited. 
[13] J. Guckenheimer, Isochrons and phaseless sets, J. Math. Biol., 1 (1974/75), pp. 259-273.

[14] À. HARo, Automatic Differentiation Tools in Computational Dynamical Systems, manuscript, http:// www.maia.ub.es/dsg/2008/ (2008).

[15] À. Haro And R. DE la Llave, A parameterization method for the computation of invariant tori and their whiskers in quasi-periodic maps: Numerical algorithms, Discrete Contin. Dyn. Syst. Ser. B, 6 (2006), pp. 1261-1300.

[16] M. W. Hirsch And C. C. Pugh, Stable manifolds and hyperbolic sets, in Global Analysis (Berkeley, CA, 1968), AMS, Providence, RI, 1970, pp. 133-163.

[17] E. M. Izhikevich, Dynamical Systems in Neuroscience: The Geometry of Excitability and Bursting, Computational Neuroscience, MIT Press, Cambridge, MA, 2007.

[18] À. Jorba And M. Zou, A software package for the numerical integration of ODEs by means of high-order Taylor methods, Experiment. Math., 14 (2005), pp. 99-117.

[19] B. Krauskopf And H. Osinga, Growing $1 D$ and quasi-2D unstable manifolds of maps, J. Comput. Phys., 146 (1998), pp. 404-419.

[20] C. Morris And H. LeCAR, Voltage oscillations in the barnacle giant muscle fiber, Biophys. J., 35 (1981), pp. 193-213.

[21] P. J. Olver, Applications of Lie Groups to Differential Equations, 2nd ed., Grad. Texts in Math. 107, Springer-Verlag, New York, 1993.

[22] S. OpRisan And C. CANAvier, The influence of limit cycle topology on the phase resetting curve, Neural Comp., 14 (2002), pp. 1027-1057.

[23] A. Ptitsyn, S. Zvonic, And J. Gimble, Digital signal processing reveals circadian baseline oscillation in majority of mammalian genes, PLoS Comput. Biol., 3 (2007), pp. 1108-1114.

[24] J. Rinzel and G. Ermentrout, Analysis of neural excitability and oscillations, in Methods in Neural Modeling, C. Koch and I. Segev, eds., MIT Press, Cambridge, MA, 1998, pp. 135-169.

[25] M. SAbatini, Isochronous sections via normalizers, Internat. J. Bifur. Chaos Appl. Sci. Engrg., 15 (2005), pp. 3031-3037.

[26] E. SELKov, On the mechanism of single-frequency self-oscillations in glycolysis. A simple kinetic model, European J. Biochem., 4 (1968), pp. 79-86.

[27] C. Simó, On the analytical and numerical approximation of invariant manifolds, in Les Méthodes Modernes de la Méchanique Céleste, D. Benest and C. Froeschlé, eds., Editions Frontières, Gif-sur-Yvette, France, 1990, pp. 285-329.

[28] A. T. Winfree, Patterns of phase compromise in biological cycles, J. Math. Biol., 1 (1974/75), pp. 73-95.

Copyright ( $\odot$ by SIAM. Unauthorized reproduction of this article is prohibited. 